normale Tracheïden des jungen Ueberwallungsholzes. Die hier zahlreich auftretenden Harzgänge scheinen mehr die Nachwirkung des Reizes zu sein, als ein Schutzmittel der Pflanze.

Ausser dem vorstehend Angeführten ergaben sich im Laufe der Arbeit noch eine Menge von Einzelheiten, welche, an sich meist unbedeutend und belanglos, in ihrer Gesamtheit doch einige aufklärende Streiflichter werfen auf die interessanten Vorgänge, die sich bei der Bildung der Harzgallen abspielen. Die eigentliche, ausführliche and auch die Litteratur eingehend berücksichtigende Arbeit wird demnächst in der forstlich-naturwissenschaftlichen Zeitschrift in München erscheinen. Es sind derselben der leichteren und schnelleren Uebersicht halber 12, meist schematische Textfiguren beigegeben und eine Tafel mit 7 autotypischen Reproduktionen nach von mir aufgenommenen Photographien von Harzgallen-Querschnitten.

Mitteilungen aus dem pharmazeutisch-chemischen Institut der Universität Marburg.

\title{
Ueber die Alkaloide der Lupinensamen.
}

\author{
Von Ernst Schmidt.
}

(Zweite Mitteilung ${ }^{1}$ ).

\section{Ueber das Lupinin und das Lupinidin der gelben Lupine.}

Von Dr. L. Berend.

(Eingegangen den 15. IV. 1897).

Die Samen der gelben Lupine, Lupinus luteus, sind im Laufe der Jahre bereits von verschiedenen Chemikern einer Untersuchung unterzogen worden, ohne dass es jedoch bisher gelungen ist, namentlich die alkaloidartigen Bestandteile derselben zuverlässig zu charakterisieren. Schon die, die Elementarzusammensetzung jener Basen betreffenden Daten weichen in einer so überraschenden Weise von einander $a^{2}$ ), dass es den Anschein gewinnt, als sei die Isolierung und Reindarstellung derselben mit ganz besonderen Schwierigkeiten verknüpft.

1) Vgl. dieses Archiv 1897, S. 192.

2) Vgl. daselbst S. 196. 
Zur Aufklärung dieser Widersprüche, welche in der Litteratur über die Alkaloide der Samen der gelben Lupine obwalten, unternahm ich auf Veranlassung meines hochverehrten Lehrers, des Herrn Geh. Rat Prof. Dr. E. Schmidt die Ausführung der vorliegenden Arbeit.

Darstellung der Lupinenalkaloide.

Behufs Darstellung dieser Basen verfuhr ich nach der von Baumert') erwähnten, zunächst von Liebscher ${ }^{2}$ ) angewendeten Methode, wie folgt:

Ein Zentner grob geschrotene Samen der gelben Lupine wurde in drei grösseren Dekantiertöpfen mit salzsäurehaltigem Alkohol derart ausgezogen, dass, nachdem der 1. Auszug abgezogen war, dieser Topf mit dem Auszug des $\mathrm{zweiten}$, der zweite mit dem Auszug des dritten, letzterer endlich mit frischem Alkohol beschickt wurde. Somit war nach wiederholtem gleichen Behandeln der erste Topf mit dem am meisten angereicherten Auszug beschickt. Die Auszüge wurden dann nacheinander abdestilliert, die Samen von neuem mit salzsäurehaltigem Alkohol übergossen und dies Verfahren etwa zehn Mal in derselben Weise wiederholt. Der im Destillationsgefäss gebliebene Extraktrückstand wurde in einer Abdampfschale mehrere Male mit Wasser erhitzt, wodurch das reichlich vorhandene Fett sich in zusammenhängenden Massen abschied. Nunmehr wurde die Flüssigkeit filtriert, eingedampft, mit Natronlauge alkalisch gemacht und im Scheidetrichter mit Chloroform die Alkaloide ausgeschüttelt. Später benutzte ich zu letzterem Zweck Aether, da das Chloroform sehr viele Verunreinigungen harzartiger Natur mit aufnimmt, wodurch die weitere Reinigung der Basen sehr erschwert wird.

Nach dem Abdestillieren des Chloroforms, bezw. Aethers blieb eine dicke, extraktähnliche Masse zurück, die ich bis zum vollständigen Verschwinden des betreffenden Lösungsmittels erwärmte und nach dem Erkalten in den Exsiccator setzte. Sehr bald, verschiedentlich schon nach einigen Stunden, zeigte sich Krystallbildung, die schnell fortschritt. Die Krystalle wurden abgesogen, zwischen Thonplatten und dann zwischen Filtrierpapier getrocknet, das Filtrat dagegen zur weiteren Krystallisation eingedampft und dann bei Seite gesetzt. Erfolgte keine Abscheidung von Krystallen mehr, so schritt ich zur weiteren Trennung der Alkaloide nach dem von Baumert herrührenden Verfahren, welches auf der Unlöslichkeit des schwefelsauren Salzes der flüssigen Base, des Lupinidins, in absolutem Alkohol beruht. Später bediente ich mich $z \mathbf{u}$ diesem $Z_{\text {weck }}$ des von mir zuerst dargestellten und analysierten Lupinidinquecksilberchlorids, welches in kaltem Wasser nahezu unlöslich ist. Letztere Methode werde ich später näher erörtern.

Zur Abscheidung des Lupinidinsulfates, welche nicht immer glatt verläuft und sehr von Zufälligkeiten abhängt, gelangte ich nach mebreren Versuchen am zweckmässigsten in nachstehender Weise.

Die zum Teil vom Lupinin befreite Extraktmenge rührte ich mit Schwefelsäure, im Verhältnis von 1:1 mit Wasser gemischt, an und gab

1) Habilitationsschrift Halle 1881.

2) Berichte des landwirtsch. Instituts Halle, II. 
dann absoluten Alkohol in starkem Ueberschuss unter kräftigem Umrühren zu. Hierbei trat alsbald die Abscheidung eines gelblich gefärbten Krystallmehles von Lupinidinsulfat ein, welches abgesogen und das Filtrat davon eingedampft wurde. Bei öfterer Wiederholung dieses Verfahrens gelang es, die grösste Menge des Lupinidins als Lupinidinsulfat zur Abscheidung zu bringen. Den Rest des eingedampften Extraktrückstandes, sowie die späteren Rückstände vom Ausschütteln mit Aether bebandelte ich folgendermafsen. Nach dem Versetzen mit Salzsäure gab ich konzentrierte Quecksilberchloridlösung so lange zan, als noch ein Niederschlag entstand, liess denselben absetzen und brachte ibn auf ein Saugfilter. Das Filtrat davon verdünnte ich mit Wasser und fällte das Quecksilber durch Schwefelwasserstoff aus. Nach dem Entfernen des Quecksilbersulfids wurde die abfiltrierte Flüssigkeit. welche, wie die Versuche ergeben hatten, nur noch salzsaures Lupinin enthielt, eingedampft, mit Natronlauge alkalisch gemacht und von neuem mit Aether ausgeschüttelt. Das durch Quecksilberchlorid ausgeschiedene und abgesogene Lupinidinquecksilberchlorid wurde dagegen mit salzsäurehaltigem Wasser angeschüttelt, mit Schwefelwasserstoff zerlegt und das abgeschiedene Quecksilbersulfid von dem in Lüsung befindlichen Lupinidinchlorid abfiltriert.

Es ist diese Methode der Trennung der Lupinenbasen den bisher bekannten entschieden vorzuziehen. Bietet dieselbe schon die Möglichkeit, das Lupinidin fast quantitativ als Lupinidinquecksilberchlorid abzuscheiden, so dürfte in zweiter Linie, als wesentlicher Vorzug, gegenüber der Beyer schen Platinchloridtrennung, der bedeutend geringere Wert des Quecksilberchlorids in Betracht kommen.

Nachdem ich nunmehr eine grössere Menge von Lupinin zur Verfügung hatte, begann ich mit der Reindarstellung desselben, was ich durch mehrfaches Umkrystallisieren aus Petroläther leicht ermöglichte.

Auf die Reinigung des Lupinidins bezw. seiner Salze komme ich weiter unten, insbesondere bei den einzelnen Derivaten desselben, zurück.

\section{A. Das Lupinin: $\mathrm{C}^{21} \mathrm{H}^{40} \mathrm{~N}^{2} \mathrm{O}^{2}$.}

Wie oben erwähnt, benutzte ich zum Umkrystallisieren der aus den Chloroform- bezw. Aether-Ausschüttelungen erhaltenen gelb gefärbten Krystalle des Lupinins Petroläther. Dabei blieb schon beim ersten Mal der grösste Teil von Unreinigkeiten in Gestalt harzartiger, brauner Massen zurück, und nach mehrmaliger Wiederholung dieser Manipulation erhielt ich vollkommen farbloses Material. Die Krystallform schien, wie auch Baumert erwähnt, je nach der Konzentration der Lösung eine verschiedene zu sein. Ganz besonders gut ausgebildet erhielt ich einmal ca. $4 \mathrm{~cm}$ lange, farblose, durchsichtige Nadeln, die, nachdem sie zwischen Filtrierpapier getrocknet, durchaus luftbeständig blieben. Im Uebrigen schienen meist kleine, rhombische, wohl auch tafelförmige Krystalle vorherrschend zu sein. Bezüglich der Beobachtung Siewerts, über den Unterschied der Löslichkeit des Lupinins in kaltem und warmem Wasser, möchte ich erwähnen, 
dass bei zunehmender Temperaturerhöhung eine stets stärker werdende 'Trübung erfolgt, die beim Kochen zur teilweisen Abscheidung des Lupinins in gelben durchsichtigen Tropfen führt. Das darüber stehende Wasser bleibt klar, und nach dem, unter Umschütteln erfolgten Erkalten, tritt wieder vollständige Lösung des abgeschiedenen Lupinins ein.

Am leichtesten löslich fand ich das Lupinin in Aether und Chloroform, ebenso, nur etwas langsamer, in Alkohol, Wasser und Petroläther.

Den Schmelzpunkt fand ich bei mehreren Proben meines aus Petroläther umkrystallisierten Alkaloids zwischen 67 und $68^{\circ}$ liegend, also den Angaben von Baumert, bezw. Liebscher entsprechend.

Von dem mir nunmehr zur Verfügung stehenden, durchaus den Stempel unbedingter Reinheit tragenden Lupinin führte ich, zur Feststellung der empierischen Zusammensetzung desselben, folgende Elementaranalysen aus, von denen die ersten vier im offenen, die letzten im Schnabelrohr vorgenommen wurden.

I. $0,2632 \mathrm{~g}$ ergaben $0,6867 \mathrm{~g} \mathrm{CO}^{2}$ und $0,2568 \mathrm{~g} \mathrm{H} 2 \mathrm{O}$.

II. $0,2269 \mathrm{~g}$ ergaben $0,5957 \mathrm{~g} \mathrm{CO}^{2}$ und $0,2186 \mathrm{~g} \mathrm{H}^{2} \mathrm{O}$.

III. $0,238 \mathrm{~g}$ ergaben $0,621 \mathrm{~g} \mathrm{CO} \mathrm{CO}^{2}$ und $0,24 \mathrm{~g} \mathrm{H}^{2} \mathrm{O}$.

IV. $0,2051 \mathrm{~g}$ ergaben $0,5344 \mathrm{~g} \mathrm{\textrm {CO } ^ { 2 }}$ und $0,2062 \mathrm{~g} \mathrm{H} 20$.

Diese Analysen ergaben folgende Zahlen für Kohlenstofi und Wasserstoff:

$\begin{array}{ccccc} & \text { I. } & \text { II. } & \text { III. } & \text { IV. } \\ \text { C. } & 71,04 & 71,56 & 71,00 & 71,03 \\ \text { H. } & 10,8 & 10,66 & 11,17 & 11,16\end{array}$

Im Schnabelrohr ausgeführte Analysen:

I. $0,374 \mathrm{~g}$ ergaben $0,9818 \mathrm{~g} \mathrm{CO}^{2}$ und $0,3958 \mathrm{~g} \mathrm{H}^{2} \mathrm{O}$.

II. $0,3486 \mathrm{~g}$ ergaben $0,9122 \mathrm{~g} \mathrm{CO}^{2}$ und $0,3721 \mathrm{~g} \mathrm{H}^{2} \mathrm{O}$.

III. $0,4658 \mathrm{~g}$ ergaben $1,2166 \mathrm{~g} \mathrm{CO} \mathrm{CO}^{2}$ und $0,4906 \mathrm{~g} \mathrm{H}^{2} \mathrm{O}$.

IV. $0,1328 \mathrm{~g}$ ergaben $0,3484 \mathrm{~g} \mathrm{CO}^{2}$ und $0,1403 \mathrm{~g} \mathrm{H}^{2} \mathrm{O}$.

Die Werte, welche diese Analysen ergaben, sind:

$\begin{array}{ccccc} & \text { I. } & \text { II. } & \text { III. } & \text { IV. } \\ \text { C. } & 71,57 & 71,34 & 71,23 & 71,54 \\ \text { H. } & 11,73 & 11,81 & 11,70 & 11,67\end{array}$

Eine nach der Dumas'schen Methode zur Ausführung gebrachte Stickstoff-Bestimmung lieferte folgendes Resultat:

$0,1609 \mathrm{~g}$ Lupinin gaben $16,5 \mathrm{ccm} \mathrm{N}$. bei $15^{\circ} \mathrm{C}$. und $750 \mathrm{~mm}$ Barometerstand, entspr. $0,0133559 \mathrm{~g} \mathrm{~N} .=8,3 \% \mathrm{~N}$.

Vergleiche ich die gefundenen Werte unter einander, so ergaben die Analysen im geschlossenen Rohr die brauchbarsten Resultate, und dürfte daher bei der sehr flüchtigen und zugleich schwer verbrennbaren Substanz letzterer Methode der Vorzug zu geben sein. Die von mir 
zu den vorerwähnten Analysen verwendete Substanz war stets zum konstanten Gewicht über Schwefelsäure getrocknet worden; bei allen Proben war hierbei ein kaum merkbarer Gewichtsverlust zu konstatieren.

Die obigen Zahlen führen, im Einklang mit den Angaben Baumerts zu der Formel: $\mathrm{C}^{21} \mathrm{H}^{40} \mathrm{~N}^{2} \mathrm{O}^{2}$.

Für die von den früheren Autoren für das Lupinin aufgestellten Formeln berechnen sich folgende Werte:

$\begin{array}{llrrr}\text { Siewert } & \mathrm{C}^{10} \mathrm{H}^{21} \mathrm{NO}-\mathrm{C} 70,17 & \mathrm{H} & 12,28 & \mathrm{~N} 8,18 \\ \text { Beyer } & \mathrm{C}^{10} \mathrm{H}^{23} \mathrm{NO}^{2}- & 63,49 & 12,16 & 7,41 \\ \text { Schulz } & \mathrm{C}^{10} \mathrm{H}^{21} \mathrm{NO}^{2}- & 64,17 & 11,23 & 7,48 \\ \text { Liebscher } & \mathrm{C}^{10} \mathrm{H}^{20} \mathrm{NO}- & 70,59 & 11,76 & 8,23\end{array}$

Gefunden durch Analyse der freien Base:

$\begin{array}{lccc} & \mathrm{C} & \mathrm{H} & \mathrm{N} \\ \text { Siewert } & 70,01 & 11,9 & - \\ \text { Schulz } & 64,06 & 11,31 & 7,43 \\ \text { Liebscher } & 70,85 & 11,73 & -\end{array}$

Von Beyer liegt eine Analyse des Lupinins selbst nicht vor. Gefunden und berechnet für die Formel: $\mathrm{C}^{21} \mathrm{H}^{40} \mathrm{~N}^{2} \mathrm{O}^{2}$.

$\begin{array}{ccc}\text { Baumert } & \text { Berend } & \text { Berechnet } \\ \mathrm{C}-71,51 & 71,42 & 71,59 \\ \mathrm{H}-11,61 & 11,72 & 11,35 \\ \mathrm{~N}-8,10 & 8,3 & 7,95\end{array}$

$$
\text { Salze des Lupinins. }
$$

Bezüglich der Salze des Lupinins sind, bis auf die Untersuchungen Baumerts, nur sehr dürftige Angaben in der Litteratur zu finden; nur das Platindoppelsalz hat seitens der früheren Forscher ein eingehenderes Studium erfahren. Zur Bestätigung obiger Formel habe ich einige Salze des Lupinins dargestellt und von Neuem untersucht.

Lupininplatinchlorid: $\mathrm{C}^{21} \mathrm{H}^{40} \mathrm{~N}^{2} \mathrm{O}^{2} \mathrm{Pt} \mathrm{Cl}^{4} \cdot 2 \mathrm{HCl}+\mathrm{H}^{2} \mathrm{O}$. Dieses Salz ist nach Angaben von Beyer, ${ }^{1}$ ) Siewert, ${ }^{2}$ ) Schulz, ${ }^{3}$ ) und Baumert ${ }^{4}$ ) leicht rein zu erhalten, da es das einzige Platinsalz der Alkaloide der gelben Lupinen ist, welches sich leicht in Alkohol löst. Beyer gründete sogar auf diese Eigenschaft eine Trennungsmethode der beiden Alkaloide der gelben Lupine. Siewert scheint kein reines Salz in Händen gehabt zu haben, wenigstens weist die leichte

1) Landwirtschaftl. Versuchsstation 14 (1876).

2) Ibidem 12, 306.

3) Landwirtschaftl. Jahrbücher 1879.

4) L. C. 
Zersetzbarkeit des von ihm gewonnenen Doppelsalzes bei ca. $100^{\circ}$ darauf hin. Schul z erhielt das Lupininplatinchlorid in grossen, glänzenden, rubinroten, dem klinorhombischem System angehörenden Krystallen.

B a u mert stellte das Salz dar, indem er eine salzsaure, wässerige oder alkoholische Lösung des Lupinins mit Platinchlorid vermischt und im Vakuum über Aetzkali langsam verdunsten liess. Einmal umkrystallisiert, erhielt er dieselben vollkommen rein und in der von Schulz schon beschriebenen Krystallform. Ich verfuhr zur Darstellung dieses Doppelsalzes so, dass ich Lupinin in wenig Wasser löste, diese Lösung mit Salzsäure neutralisierte und dann eine berechnete Menge 10\% Platinchloridlösung, sowie etwas Alkohol, zugab. Schon nach einem Tage zeigten sich vereinzelte Krystalle, die sich schnell vermehrten. Der Schmelzpunkt dieses Präparates lag bei 163-164 ${ }^{\circ}$. Die Analyse desselben ergab folgende Daten:

I. $0,25 \mathrm{~g}$ ergaben $0,0054 \mathrm{~g}$ Wasserverlust bei $105^{\circ}$. $0,2446 \mathrm{~g}$ wasserfreies Salz ergab $0,063 \mathrm{Pt}$.

II. $0,1595 \mathrm{~g}$ ergaben $0,0039 \mathrm{~g}$ Wasserverlust bei $105^{\circ}$. $0,1156 \mathrm{~g}$ wasserfreies Salz ergaben $0,0398 \mathrm{Pt}$.

III. $0,310 \mathrm{~g}$ ergaben $0,0072 \mathrm{~g}$ Wasserverlust bei $105^{\circ}$. $0,3028 \mathrm{~g}$ wasserfreies Salz ergaben $0,366 \mathrm{~g} \mathrm{CO}^{2}$ und $0,1584 \mathrm{~g} \mathrm{H} \mathrm{H}^{2} \mathrm{O}$.

$$
\begin{array}{rrrr}
\multicolumn{5}{c}{\text { Gefunden: }} \\
& \text { I. } & \text { II. } & \text { III. } \\
\text { C } & - & - & 32,96 \\
\text { H } & - & - & 5,81 \\
\mathrm{Pt} & 25,57 & 25,75 & - \\
\text { H } 20 & 2,16 & 2,44 & 2,32
\end{array}
$$

Diese Werte kommen den von Baumert gefundenen und für die von ihm aufgestellte Formel des Lupininplatinchlorids berechneten sehr nahe:

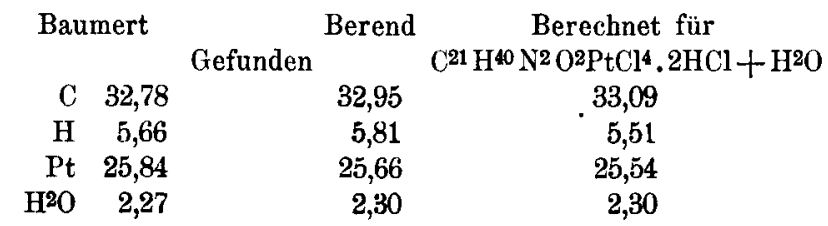

Lupiningoldchlorid: $\mathrm{C}^{21} \mathrm{H}^{40} \mathrm{~N}^{2} \mathrm{O}^{2} 2 \mathrm{HCl} \cdot 2 \mathrm{AuCl}{ }^{3}$. Dieses Salz wird zuerst von Baumert erwähnt, der dasselbe aus salzsaurem Lupinin durch Fällung in wässeriger Lösung erhielt. Aus heissem Wasser umkrystallisiert, schied es sich in nadedelförmigen, federartig an einander gereihten Krystallen aus. Das von mir in ähnlicher Weise dargestellte Lupiningoldchlorid krystallisierte in schönen, glänzenden Nädelchen, die sich sternförmig zusammenlagerten. Nach 
mehrmaligem Umkrystallisieren erhielt ich auch einige grössere, rhombische Krystalle, die vollkommen durchsichtig waren. Den Schmelzpunkt dieser letzteren fand ich bei $196-197^{\circ}$ liegend, während Baumert ca. $195^{\circ}$ angiebt. Das Lupiningoldchlorid bleibt bei $100^{\circ}$ unverändert, dagegen tärbt es sich bei $105^{\circ}$ dunkler und bräunt sich bei weiterer Temperaturerhöhung.

I. 0,1096 g Substanz ergaben 6,0418 g Au.

II. 0,246 g Substanz ergaben $0,0936 \mathrm{~g}$ Au.

III. 0,2794 g Substanz ergaben bei der Elementaranalyse im Bleichromatrohr $0,2485 \mathrm{~g} \mathrm{CO}^{2}$ und $0,1069 \mathrm{~g} \mathrm{H}^{20}$.

I.

$\begin{array}{rc}\mathrm{Au} & 38,14 \\ \mathrm{C} & - \\ \mathrm{H} & -\end{array}$

Baumert Gefunden

$\begin{array}{rr}\mathrm{Au} & 38,18 \\ \mathrm{C} & 24,25 \\ \mathrm{H} & 4,21 \\ \mathrm{Cl} & 27,43\end{array}$
II. 38,04

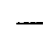

Berend

38,11

24,23

4,24
III.

24,23

4,24

Berechnet für $\mathrm{C}^{21} \mathrm{H}^{40} \mathrm{~N}^{2} \mathrm{O}^{2} 2 \mathrm{H} \mathrm{Cl} 2 \mathrm{AuCl}^{3}$

$$
\begin{array}{r}
38,08 \\
24,45 \\
4,07 \\
27,55
\end{array}
$$

Zur weiteren Bestätigung der Formel $\mathrm{C}^{21} \mathrm{H}^{40} \mathrm{~N}^{2} \mathrm{O}^{2}$ löste ich $0,1 \mathrm{~g}$ reines Lupinin in $100 \mathrm{ccm} \mathrm{1/100} \mathrm{Norm.} \mathrm{Schwefelsäure} \mathrm{auf,} \mathrm{gab} \mathrm{etwas}$ atherische Jodeosinlösung zu und titrierte die nicht durch das Lupinin gebundene Schwefelsäure mit $1 / 100$ Norm. Kalilauge zurück. Verbrauch† wurden $43 \mathrm{~cm} \mathrm{1/100} \mathrm{Norm.} \mathrm{Kalilauge.} \mathrm{Durch} \mathrm{das} \mathrm{Lupinin} \mathrm{wurden} \mathrm{alse}$ $57 \mathrm{ccm} 1 / 100$ Norm. Schwefelsäure gebunden, entsprechend einer Menge von $0,0279 \mathrm{~g} \mathrm{H}^{2} \mathrm{SO}^{4}$. Zur Bildung von Lupininsulfat $\mathrm{C}^{21} \mathrm{H}^{40} \mathrm{~N}^{2} \mathrm{O}^{2}$. $\mathrm{H}^{2} \mathrm{SO}^{4}$ sind für $0,1 \mathrm{~g}$ Lupinin genau $0,0279 \mathrm{~g} \mathrm{H}^{2} \mathrm{SO}^{4}$ erforderlich. Es dürfte somit auch dieses Resultat die Richtigkeit der Formel $\mathrm{C}^{21} \mathrm{H}^{40} \mathrm{~N}^{2} \mathrm{O}^{2}$ für das Lupinin, bezw. $\mathrm{C}^{21} \mathrm{H}^{40} \mathrm{~N}^{2} \mathrm{O}^{2} \cdot \mathrm{H}^{2} \mathrm{SO}^{4}$ für das neutrale Lupininsulfat beweisen.

Das optische Verhalten des Lupinins und seiner Salze.

Baumert giebt nur an, dass dieses Alkaloid und seine Salze den polarisierten Lichtstrahl nach links ablenken. Die Ergebnisse meiner mit dem Laurent'schen Apparat ausgeführten Untersuchungen stehen hiermit im Einklang. Je nach der Konzentration der Lösungen ändert sich das spezifische Drehungsvermögen:

I. $0,79 \mathrm{~g}$ reines Lupinin in $25 \mathrm{~g}$ Wasser gelöst zeigten im Durchschnitt eine Ablenkung von $-1^{0} 50^{4}$ im $220 \mathrm{~mm}$-Rohre bei ca. $18^{0}$.

$$
[\alpha] \mathrm{D}=\frac{11000}{1,01 \cdot 2,2 \cdot 3,16}=-26^{0} \text {. }
$$


II. $0,395 \mathrm{~g}$ reines Lupinin in $25 \mathrm{~g} \mathrm{H}^{2} \mathrm{O}$ gelöst zeigten im Durchschnitt eine Ablenkung von $-50^{\prime}$ im $220 \mathrm{~mm}$-Rohre.

$$
[\alpha] \mathrm{D}=\frac{5000}{1,01 \cdot 2,2 \cdot 1,58}=-23^{0} 7^{\prime} .
$$

III. $0,1986 \mathrm{~g}$ reines Lupinin in $21,05 \mathrm{~g} \mathrm{H} \mathrm{H}^{2} \mathrm{O}$ gelöst zeigten im Durchschnitt eine Ablenkung von -25' im 220 mm-Rohre.

$$
\text { [0. } \mathrm{D}=\frac{2500}{1 \cdot 2,2 \cdot 0,95}=-20^{\circ} \text {. }
$$

I.

Lös. $3,16 \%$

$-26^{\circ}$
II.

$1,58 \%$

$-2307$
III.

$0,95 \%$

$-200$

\section{Salzsaures Lupinin.}

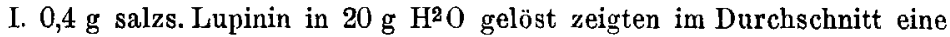
Ablenkung von -170 .

$$
[\alpha] \mathrm{D}=\frac{1700}{1,01 \cdot 1 \cdot 2}=-14^{0} \text { in } 100 \text { mm-Rohre. }
$$

Das spez. Drehungsvermögen des salzsauren Lupinins beträgt also in $2 \%$ Lösung: $-14^{0}$.

Einwirkung von Brom auf Lupinin.

Da es Davis ${ }^{1}$ ) gelungen war, das Hydrochlorid des RechtsJ Jupanins durch Brom in zwei neue Basen $\mathrm{C}^{8} \mathrm{H}^{15} \mathrm{NO}$ und $\mathrm{C}^{7} \mathrm{H}^{11} \mathrm{NO}$ zu zerlegen, versuchte auch ich eine ähnliche Einwirkung des Broms auf das Lupinin zu erzielen. Zu diesem Zweck löste ich ca. $1 \mathrm{~g}$ Lupinin in wenig Alkohol, neutralisierte mit Bromwasserstoffsäure, gab alkoholische Bromlösung (1:1) im Ueberschuss zu und liess diese Lösung über Schwefelsäure und Aetzkalk verdunsten. Nach einigen 'Tagen zeigten sich strahlenförmig aneinandergelagerte Krystalle, die auf einem Filter gesammelt und mit Aether-Alkohol gewaschen wurden. Dieselben waren leicht löslich in Alkohol und Wasser; sie erwiesen sich luftbeständig.

$0,1716 \mathrm{~g}$ Substanz verloren bei $100^{\circ} 0,0008 \mathrm{~g}$ an Gewicht, welche Differenz wohl nur auf anhaftende Feuchtigkeit zurückzuführen sein dürfte. $0,1708 \mathrm{~g}$ der bei $100^{\circ}$ konstant gebliebenen Substanz gaben 0,1298 $\mathrm{g} \mathrm{Ag} \mathrm{Br}$, entsprechend $32,32 \% \mathrm{Br}$.

Aus diesen Daten scheint hervorzugehen, dass das vorliegende Salz nur ein nicht ganz reines bromwasserstoffsaures Lupinin von der Formel $\mathrm{C}^{21} \mathrm{H}^{40} \mathrm{~N}^{2} \mathrm{O}^{2} \cdot 2 \mathrm{HBr}$ war:

Berechnet für $\mathrm{C}^{21} \mathrm{H}^{40} \mathrm{~N}^{2} \mathrm{O}^{3} .2 \mathrm{HBr}$ $31,12 \% \mathrm{Br}$
Gefunden $32,32 \% \mathrm{Br}$.

1) Dieses Archiv 1897, 236. 
Um einen weiteren Einblick in die chemische Natur dieses Bromeinwirkungsproduktes zu gewinnen, setzte ich die Lösung desselben mit Chlorsilber um und führte einen Teil derselben in das Gold-, einen anderen Teil in das Platindoppelsalz über. Auf Zusatz von Goldchloridlösung entstand ein flockiger Niederschlag, der umkrystallisiert sich in feinen, nadelförmigen Krystallen ausschied. Dieselben schmolzen bei $194-195^{\circ}$ und verloren bei $100^{\circ}$ kaum an Gewicht.

$0,168 \mathrm{~g}$ des bei $100^{\circ}$ getrockneten Salzes gaben $0,064 \mathrm{~g}$ Au entsprechend $38,09 \% \mathrm{Au}$.

Dass es sich bei dem analysierten Doppelsalze nur um Lupiningoldchlorid handelte, also eine Einwirkung des Broms in der erhofften Weise nicht stattgefunden hatte, dürfte durch den Goldgehalt sowohl, als auch den Schmelzpunkt bewiesen sein.

$\begin{array}{cc}\text { Gefunden } & \text { Berechnet für } \mathrm{C}^{21} \mathrm{H}^{40} \mathrm{~N}^{2} \mathrm{O}^{2} \cdot 2 \mathrm{HCl} 2 \mathrm{Au} \mathrm{Cl}^{3} \\ 38,09 \% \mathrm{Au} & 38,08 \% \mathrm{Au} .\end{array}$

Ebenso zeigte auch das in wohlausgebildeten Krystallen erhaltene Platinsalz einen dem Lupininplatinchlorid entsprechenden Wasser- und Platingehalt, denn:

$0,1588 \mathrm{~g}$ Substanz verloren bei etwas über $10000,0038 \mathrm{~g} \mathrm{H}^{2} \mathrm{O}$.

$0,155 \mathrm{~g}$ wasserfreie Substanz gaben $0,039 \mathrm{~g}$ Pt.

$\begin{array}{cc}\text { Gefunden } & \text { Berechnet für } \mathrm{C}^{21} \mathrm{H}^{40} \mathrm{~N}^{2} \mathrm{O}^{2} \mathrm{PtCl}^{4} .2 \mathrm{HCl}+\mathrm{H}^{2} \mathrm{O} \\ 2,38 \% \mathrm{H}^{2} \mathrm{O} & 2,30 \% \mathrm{H}^{2} \mathrm{O} \\ 25,35 \% \mathrm{Pt} & 25,54 \% \mathrm{Pt} .\end{array}$

Da somit auf diese Weise das Lupinin keine Veränderung erfahren hatte, nahm ich den Versuch der Bromierung in etwas anderer Form vor.

Eine Druckflasche beschickte ich mit $1 \mathrm{~g}$ Lupinin, gelöst in Eisessig, und ca. $2 \mathrm{~g}$ reinem Brom. Nach etwa dreistündigem Erhitzen im Dampfbade liess ieh erkalten und behandelte die intensiv rot gefärbte Flüssigkeit mit Chlorsilber im Ueberschuss. Nach dem Filtrieren und Eindampfen der klaren Flüssigkeit, verwandelte ich letztere in das entsprechende Goldsalz. Dasselbe setzte sich sofort als voluminöser, flockiger Niederschlag zu Boden und liess sich leicht umkrystallisieren. Die Krystalle zeigten die dem Lupiningoldchlorid entsprechende, nadelförmige Gestalt. Dass es sich thatsächlich um letzteres Salz handelte, zeigte die Schmelzpunkts- und Goldbestimmung. Schmelzpunkt 195, $5^{0}$.

I. $0,212 \mathrm{~g}$ bei $100^{\circ}$ getrockneter Substanz erwiesen sich an Gewicht konstant und gaben $0,0811 \mathrm{~g} \mathrm{Au}$.

II. 0,198 bei $10 \mathrm{C}^{\circ}$ getrockneter Substanz gaben $0,0756 \mathrm{~g} \mathrm{Au}$.

Gefunden

I.

$38,25 \% \mathrm{Au}$
II.

$38,14 \% \mathrm{Au}$
Berechnet für

$\mathrm{C}^{21} \mathrm{H}^{40} \mathrm{~N}^{2} \mathrm{O}^{2} .2 \mathrm{HCl} \cdot 2 \mathrm{AuCl}^{3}$ $38,08 \% \mathrm{Au}$. 
Es war somit auch bei diesem Versuch eine Spaltung des Lupinins nicht erfolgt; ich modifizierte daher den Bromierungsversuch in nachstehender Weise:

Eine Lösung von etwa $2 \mathrm{~g}$ Lupinin in Wasser wurde solange mit Bromwasser versetzt, als sich noch eine Abscheidung eines gelblich weissen Niederschlages bemerkbar machte. Derselbe wurde abfiltriert, in Alkohol gelöst und die Lösung zum freiwilligen Verdunstenlassen beiseite gesetzt. Die von dem Niederschlag abfiltrierte Flüssigkeit dampfte ich auf ein kleines Volumen ein und stellte den erkalteten, ölig aussehenden, gelblich weissen Rückstand üher Schwefelsäure und Aetzkalk zur eventuellen Krystallisation. Beide Rückstände erstarrten zu einer hygroskopischen, weissgelblichen Krystallmasse, die weder aus Alkohol, noch Wasser umzukrystallisieren war. Die Lösung dieser Produkte wurde daher mit Chlorsilber umgesetzt und hierauf zur Darstellung der Goldsalze verwendet. Letztere resultierten nach dem Umkrystallisieren als sehr feine, gleichmässig aussehende Krystallnädelchen, welche bei $193^{\circ} \mathrm{schmolzen}$.

a. Goldsalz aus dem Rückstand der alkoholischen Lösung:

I. $0,1835 \mathrm{~g}$ bei $100^{\circ}$ getrockneten Salzes gaben $0,0702 \mathrm{~g} \mathrm{Au}$.

II. $0,346 \mathrm{~g}$ des bei $100^{\circ}$ getrockneten Salzes gaben $0,308 \mathrm{~g} \mathrm{CO}^{2}$ und $0,1361 \mathrm{~g} \mathrm{H}^{2} \mathrm{O}$.

b. Goldsalz aus dem Rückstand der wässerigen Lösung. Schmelzpunkt $193-194^{\circ}$. Bei $100^{\circ}$ war ebenfalls keine Gewichtsabnahme bernerkbar.

III. $0,2461 \mathrm{~g}$ gaben $0,0938 \mathrm{~g}$ Au.

JV. 0,211 g gaben $0,0808 \mathrm{~g}$ Au.

I.

$\mathrm{Au}$

38,25

C

$\mathrm{H}$
II.

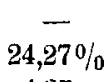

III. 4,37 ,
38,11

$-$

Auch aus diesen Daten geht hervor, dass das Lupinin durch die Einwirkung von Brom weder eine Bromierung, noch eine Spaltung erfahren haben konnte, da die beiden obenerwähnten Goldsalze nur Lupiningoldchlorid waren.

Berechnet für $\mathrm{C}^{21} \mathrm{H}^{40} \mathrm{~N}^{2} \mathrm{O}^{2} \cdot 2 \mathrm{H} \mathrm{Cl} \cdot 2 \mathrm{AuCl}^{3}$

$\begin{array}{rr}\mathrm{Au} & 38,08 \\ \mathrm{C} & 24,45 \\ \mathrm{H} & 4,07\end{array}$ Gefunden im Mittel

I. u. II.

38,25

24,27

4,37
IV. $38,29 \%$

Auch die aus dem Einwirkungsprodukte des Broms auf Lupinin regenerierte freie Base stimmte in allen ihren Eigenschaften mit dem ursprünglichen Lupinin überein. 
Einwirkung von rauchender Salzsäure, sowie von Phosphorsäureanhydrid auf Lupinin.

Schon Liebscher ${ }^{1}$ ) hatte rauchende Salzsäure bei verschiedenen Temperaturen im geschlossenen Rohr auf das Lupinin einwirken lassen. Die Analysen der bei drei verschiedenen Darstellungen erhaltenen Platinverbindungen vermochte Liebscher nicht zu deuten, da er von der unrichtigen Formel des Lupinins $\mathrm{C}^{10} \mathrm{H}^{20} \mathrm{NO}$ ausging. Erst Baumert ${ }^{2}$ ) gelang es, über das Verbalten des Lupinins zu wasserentziehenden Agentien, genauere Angaben zu machen. Als Resultat seiner Untersuchungen giebt Baumert an:

Wird salzsaures Lupinin mit Phosphorsäureanhydrid nur so hoch erhitzt, dass dabei Salzsäure nicht ausgetrieben wird, so geht das Alkaloid unter Aufnahme von drei Atomen Sauerstoff über in: Oxylupinin $\mathrm{C}^{21} \mathrm{H}^{40} \mathrm{~N}^{2} \mathrm{O}^{5}$. War das Erhitzen aber so stark, dass freie Salzsäure entsteht, oder erhitzt man direkt freies Lupinin mit Phosphorsäureanhydrid, so resultiert als Hauptprodukt: Anhydrolupinin $\mathrm{C}^{21} \mathrm{H}^{38} \mathrm{~N}^{2} \mathrm{O}$, und im letzteren Falle auch: Dianhydrolupinin $\mathrm{C}^{21} \mathrm{H}^{36} \mathrm{~N}^{2}$. Rauchende Salzsäure spaltet bei einer Tenıperatur von etwas über $200^{\circ}$ successive ein und zwei Moleküle Wasser ab und bildet so ebenfalls: Anhydrolupinin und Dianhydrolupinin. Ich habe diese Versuche Baumert's wiederholt, einesteils, um die hierbei entstehenden interessanten Körper einer weiteren Untersuchung zu unterziehen, anderenteils aber, weil ich mir keine Erklärung für das Entstehen des Oxylupinins bei der Einwirkung von Phosphorsäureanhydrid auf salzsaures Lupinin geben konnte. Ich möchte gleich hier vorausschicken, dass mir die Isolierung dieses Oxylupinins nach den Angaben Baumerts nicht gelungen ist.

Etwa $2 \mathrm{~g}$ salzsaures Lupinin wurden zu diesem Zwecke im zugeschmolzenen Rohr etwa drei Stunden lang mit Phosphorsäureanhydrid auf $180^{\circ}$ erhitzt. Die glasige Masse, in wenig Wasser gelöst und die Lösung mit Platinchloridlösung versetzt, ergab nach zweitägigem Stehen über Aetzkalk, kleine tafelförmige Kryställchen.

I. $0,1512 \mathrm{~g}$ verloren bei etwa $105^{0} 0,0033 \mathrm{~g} \mathrm{H}^{2} \mathrm{O} ; 0,1479 \mathrm{~g}$ wasserfreie Substanz ergaben $0,0376 \mathrm{~g}$ Pt.

II. $0,198 \mathrm{~g}$ verloren bei $105^{0} 0,0044 \mathrm{~g} \mathrm{H}^{2} \mathrm{O} ; 0,1936 \mathrm{~g}$ ergaben $0,232 \mathrm{~g}$ $\mathrm{CO}^{2}$ und $0,0965 \mathrm{~g} \mathrm{H}^{2} \mathrm{O}$.

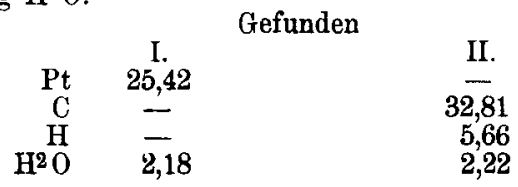

1) J. Kühns Ber. a. d landw. Inst. d. Univ. Halle. Heft II, S. 68.

2) Ann. d. Chemie 214, S. 461, Ber. d. deutsch. chem. Ges. 
Diese Werte lassen nur auf Lupininplatinchlorid und nicht auf Oxylupininplatinchlorid schliessen.

Bei einem weiteren Versuch erhitzte ich ebenfalls ca. 2 g salzsaures Lupinin mit Phosphorsäureanhydrid, jedoch ca. 6 Stunden lang, auf $180^{\circ}$. Nach dem Lösen des Röhreninhaltes in Wasser stellte ich das Goldsalz dar. Dasselbe schied sich als flockiger Niederschlag ab; auch nach dem Umkrystallisieren liess sich eine bestimmte Krystallform nicht erkennen.

I. 0,2241 $\mathrm{g}$ lufttrockener Substanz gaben $0,0865 \mathrm{~g} \mathrm{Au}$.

II. $0,2862 \mathrm{~g}$ gaben $0,2601 \mathrm{~g} \mathrm{CO}^{2}$ und $0,1112 \mathrm{~g} \mathrm{H}^{2} \mathrm{O}$.

III. $0,254 \mathrm{lg}$ gaben $0,0976 \mathrm{~g} \mathrm{Au}$.

IV. $0,181 \mathrm{~g}$ gaben $0,0696 \mathrm{~g} \mathrm{Au}$.

I.

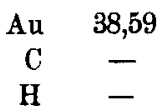

II.

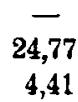

III. 38,41
IV. 38,50

Es lassen sich diese Daten nur mit dem Anhydrolupinin $\mathrm{C}^{21} \mathrm{H}^{38} \mathrm{NO}$, bez. mit dem Anhydrolupiningoldchlorid: $\mathrm{C}^{21} \mathrm{H}^{38} \mathrm{~N}^{2} \mathrm{O} 2(\mathrm{HCl} \cdot$ $\left.\mathrm{AuCl}{ }^{3}\right)$ in Einklang bringen:

Gefunden im Mittel

$$
\begin{array}{rr}
\text { Au } & 38,50 \\
\text { C } & 24,77 \\
\text { H } & 4,31
\end{array}
$$

Berechnet für $\mathrm{C}^{21} \mathrm{H}^{38} \mathrm{~N}^{2} \mathrm{O} \cdot 2\left(\mathrm{HCl} \cdot \mathrm{Au} \mathrm{Cl}^{3}\right)$

$$
\begin{array}{r}
38,79 \\
24,88 \\
3,94
\end{array}
$$

Nach der Angabe von Baumert gelingt es durch Einwirkung von rauchender Salzsäure im zugeschmolzenen Rohr auf freies Lupinin bei Temperaturen bis etwas über $200^{\circ}$, ebenfalls ein, bezw. zwei Moleküle Wasser abzuspalten und so Anhydro-, bezw. Dianhydrolupinin zu el.halten. Ich erhielt durch dreistündiges Erhitzen von rauchender Salzsäure mit freiem Lupinin auf $180^{\circ}$ jedoch nur salzsaures Lupinin. Das aus dem Einwirkungsprodukt hergestellte Goldsalz gab folgende Analysenresultate:

I. $0,282 \mathrm{~g}$ bei $100^{\circ}$ getrocknetes Salz gaben $0,1073 \mathrm{~g} \mathrm{Au}$.

II. $0,24 \mathrm{~g}$ bei $100^{\circ}$ getrocknetes Salz gaben $0,219 \mathrm{~g} \mathrm{CO} \mathrm{CO}^{2}$ und $0,096 \mathrm{H}^{2} \mathrm{O}$. Gefunden

I. II.

$\begin{array}{rcr}\mathrm{Au} & 38,0 & - \\ \mathrm{C} & - & 24,45 \\ \mathrm{H} & - & 4,27\end{array}$
Berechnet für

$\mathrm{C}^{21} \mathrm{H}^{40} \mathrm{~N}^{2} \mathrm{O}^{2} 2\left(\mathrm{H} \mathrm{Cl} \cdot \mathrm{Au} \mathrm{Cl}^{8}\right)$

38,08

24,45

4,07

Bei einem weiteren Versuch verfuhr ich so, dass ich die Mischung von Lupinin und rauchender Salzsäure einer 6 stündigen Einwirkung bei $200^{\circ}$ aussetzte. Es resultierte eine gelb gefärbte Flüssigkeit. Der Druck im Rohr war sehr gering; von einer Gasausströmung war nichts 7ll bemerken. Das durchaus gleichmässige Einwirkungsprodukt dampfte 
ich auf ein kleines Volumen ein und stellte dann daraus ein Goldsalz her. Dasselbe fiel sofort flockig aus und liess sich leicht aus salzsäurehaltigem Wasser umkrystallisieren. Die Krystalle waren sehr klein, nadelformig und durchaus einheitlich. Bei $100^{\circ}$ getrocknet verloren dieselben nichts an Gewicht.

I. $0,1991 \mathrm{~g}$ bei $100^{\circ}$ getrockneter Substanz gaben $0,0773 \mathrm{~g} \mathrm{Au}$.

II. 0,312 $\mathrm{g}$ bei $100^{\circ}$ getrockneter Substanz gaben 0,1199 $\mathrm{g} \mathrm{Au}$.

III. $0,2660 \mathrm{~g}$ bei $100^{\circ}$ getrockneter Substanz gaben $0,2411 \mathrm{~g} \mathrm{CO}{ }^{2}$ und $0,1088 \mathrm{~g} \mathrm{H}^{2} \mathrm{O}$.

Diese Analysenresultate

$\begin{array}{rccc}\mathrm{Au} & 38,82 & 38,42 & -- \\ \mathrm{C} & 24,68 & - & 24,69 \\ \mathrm{H} & - & - & 5,54\end{array}$

liessen das vorliegende Goldsalz als Anhydrolupiningoldchlorid $\mathrm{C}^{21} \mathrm{H}^{38} \mathrm{~N}^{2} \mathrm{O}$ $2\left(\mathrm{H} \mathrm{Cl} \cdot \mathrm{Au} \mathrm{Cl}^{8}\right)$ ansprechen, wie aus folgender Zusammenstellung hervorgeht:

Gefunden

im Mittel

$\begin{array}{cc}\text { Anhydrolupiningoldchl. } & \text { Dianhydrolupiningoldchl. } \\ \mathrm{C}^{21} \mathrm{H}^{38} \mathrm{~N}^{2} \mathrm{O} 2\left(\mathrm{H} \mathrm{Cl} \cdot \mathrm{Au} \mathrm{Cl}^{3}\right) & \mathrm{C}^{21} \mathrm{H}^{36} \mathrm{~N}^{2} 2\left(\mathrm{H} \mathrm{Cl} \cdot \mathrm{Au} \mathrm{Cl}^{8}\right) \\ 38,79 & 39,49 \\ 24,88 & 25,32 \\ 3,94 & 3,81\end{array}$

$\begin{array}{rr}\text { Au } & 38,62 \\ \text { C } & 24,69 \\ \text { H } & 4,54\end{array}$

In all' den obenerwähnten Versuchen war es somit nicht gelungen, das von Baumert erwähnte Oxylupinin: $\mathrm{C}^{21} \mathrm{H}^{40} \mathrm{~N}^{2} \mathrm{O}^{5}$, zu erhalten.

Dianhydrolupinin: $\mathrm{C}^{21} \mathrm{H}^{86} \mathrm{~N}$.

Diese von dem Lupinin durch den Mindergehalt von zwei Molekülen Wasser sich unterscheidende, sauerstofffreie Base erhielt Liebscher, neben Anhydrolupinin, durch Einwirkung von rauchender Salzsäure auf freies Lupinin bei verschieden hohen Temperaturen.

Baumert giebt drei Methoden an, um diese Base darzustellen:

1. Durch Einwirkung von rauchender Salzsäure auf freies Lupinin bei $150-200^{\circ}$.

2. Durch Erhitzen von Phosphorsäureanhydrid und freiem Lupinin auf $175-180^{\circ}$.

3. Durch Erhitzen salzsauren Lupinins mit Phosphorsäureanhydrid auf $180^{\circ}$.

C. E. Schulz ${ }^{1}$ glaubte zu diesem sauerstofffreien Alkaloid auch durch Schmelzen von Lupinin mit metallischem Natrium gelangen zu können, analog dem Versuch Wertheims ${ }^{2}$ ), der auf diese Weise das

1) Landw. Jahrb. 1878, S. 37.

2) Journ. f. prakt. Chemie 91. 263. 
Conhydrin $\mathrm{C}^{8} \mathrm{H}^{17} \mathrm{NO}$ in Paraconiin $\mathrm{C}^{8} \mathrm{H}^{15} \mathrm{~N}$ überführte. Baumert ${ }^{1}$ ) hat jedoch diese Ansicht von Schulz widerlegt und nachgewiesen, dass bei dem Behandeln des bei jenem Vorgang zuerst entstandenen Natriumsubstitutionsproduktes mit Wasser, freies Lupinin regeneriert wird. Mir ist die Darstellung des Dianhydrolupinins glatt nur gelungen, wenn ich freies Lupinin mit Phosphorsäureanhydrid ca. 6 Stunden im zugeschmolzenen Rohr auf $200^{\circ}$ erhitzte.

Das Einwirkungsprodukt zeigte sich als eine glasige, harte Masse, die mässig leicht in Wasser löslich war. Nach dem Filtrieren, Eindampfen und Ansäuern der wässerigen Lösung mit Salzsäure, stellte ich sowohl das Gold- als auch das Platinsalz dar. Ersteres resultierte, nach dem Umkrystallisieren aus Wasser, als dicht zusammenhängende, krystallinische Masse. Eine ausgesprochene Krystallform war, der Kleinheit der Krystalle wegen, nicht zu konstatieren.

Der Schmelzpunkt lag bei $150^{\circ}$. Ein Verlust an Krystallwasser liess sich durch Trocknen bei $100^{\circ}$ nicht feststellen.

I. $0,1818 \mathrm{~g}$ bei $100^{\circ}$ getrockneter Substanz gaben $0,073 \mathrm{~g} \mathrm{Au}$.

II. $0,2102 \mathrm{~g}$ bei $100^{\circ}$ getrockneter Substanz gaben $0,0825 \mathrm{~g}$ Au.

III. $0,2476 \mathrm{~g}$ bei $100^{\circ}$ getrocknet gaben bei der Elementaranalyse im Bleichromatrohre 0,2295 $\mathrm{g} \mathrm{C} \mathrm{O}^{2}$ und $0,0822 \mathrm{~g} \mathrm{H}^{2} \mathrm{O}$.

\begin{tabular}{rcccc} 
& \multicolumn{3}{c}{ Gefunden } & Berechnet für \\
& I. & II. & III. & $\mathrm{C}^{21} \mathrm{H}^{36} \mathrm{~N}^{2} 2\left(\mathrm{H} \mathrm{Cl} \cdot \mathrm{Au} \mathrm{Cl}^{3}\right)$ \\
$\mathrm{Au}$ & 39,28 & 39,24 & - & $39,49 \% \mathrm{Au}$ \\
$\mathrm{C}$ & - & - & 25,27 & 25,32 C \\
$\mathrm{H}$ & - & - & 3,68 & $3,81 \% \mathrm{H}$
\end{tabular}

Dianhydrolupininplatinchlorid: $\mathrm{C}^{21} \mathrm{H}^{36} \mathrm{~N}^{2} \cdot 2 \mathrm{H} \mathrm{Cl} \cdot \mathrm{Pt} \mathrm{Cl}^{4}$. Ich erhielt dieses Salz in Form kleiner, dunkelroter Krystalle.

$0,1962 \mathrm{~g}$ der bei etwas über $100^{\circ}$ getrockneten Substanz gaben $0,0528 \mathrm{~g} \mathrm{Pt}$.

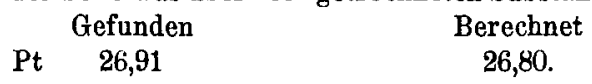

Ueber die weiteren Eigenschaften des Dianhydrolupinins s. S. 279.

Die Einwirkung von Essigsäureanhydrid auf Lupinin.

Aus dem Verhalten des Lupinins, zwei Moleküle Wasser verhältnismässig leicht abzugeben, schloss Baumert, dass diese Base ibre beiden Atome Sauerstoff in Form von alkoholischen Hydroxylen enthalte. Dies nachzuweisen, gelang Baumert durch Einführung von Acetylgruppen, und zwar sowohl durch Einwirkung von Acetylchlorid, als auch von Essigsäureanhydrid auf Lupinin. ${ }^{2}$ ) Ich kann diese Be-

1) Ber. d. deutsch. chem. Ges. 15. 631.

3) Lieb. Ann. 224. S. 313. 
obachtung nur bestätigen, und zwar durch die Analyse des bisher nicht dargestellten Golddoppelsalzes des Diacetyllupinins.

Etwa gleiche Mengen reines Lupinin und Essigsäureanhydrid wurden mit etwas entwässertem Natriumacetat ca. drei Stunden am aufrecht stehenden Kühler erhitzt. Das erhaltene, gelblich gefärbte Liquidum machte ich hierauf mit festem Natriumhydrat alkalisch und schüttelte alsdann die freie Base mit Aether aus. Den nach dem Verdunsten des Aethers gebliebenen Rückstand säuerte ich mit verdünnter Salzsänre schwach an und versetzte diese Lösung mit Goldchloridlösung. Das gebildete Goldsalz schied sich zuerst als flockiger Niederschlag ab, der aber sehr sehnell krystallinisches Gefïge annahm.

Diacetyllupiningoldchlorid: $\mathrm{C}^{21} \mathrm{H}^{38}\left(\mathrm{CH}^{3} \mathrm{CO}\right)^{2} \mathrm{~N}^{2} \mathrm{O}^{2}$ $2\left(\mathrm{HCl} \cdot \mathrm{Au} \mathrm{Cl}^{3}\right)$. Der Schmelzpunkt des lufttrocknen Salzes lag bei $120^{\circ}$. Ein Versuch, das Salz bei $100^{\circ}$, zur Bestimmung des Gehaltes an Krystallwasser, zu trocknen, wurde wegen der leichten Zersetzbarkeit desselben unterlassen; ich ging daher bei den folgenden Analysen von dem durch Stehen über Schwefelsäure und Aetzkalk konstant gewordenen Präparate aus.

I. 0,1750 g Substanz gaben 0,06022 g Au.

II. $0,250 \mathrm{~g}$ gaben bei der Elementaranalyse im Bleichromatrohr $0,246 \mathrm{~g}$ $\mathrm{CO}^{2}$ und $0,098 \mathrm{~g} \mathrm{H}^{2} \mathrm{O}$.

III. $0,312 \mathrm{~g}$ gaben bei der in gleicher Weise ausgeführten Elementaranalyse $0,3011 \mathrm{~g} \mathrm{CO}^{2}$ und $0,1298 \mathrm{~g} \mathrm{H}^{2} \mathrm{O}$.

$\begin{array}{rcccc} & & & & \text { Berechnet für } \\ \mathrm{Au} & \mathbf{3 5 , 5} & - & \text { III. } & \mathrm{C}^{21} \mathrm{H}^{38} \mathrm{~N}^{2} \mathrm{O}^{2}\left(\mathrm{CH}^{3} \mathrm{C} \mathrm{O}\right)^{2} \mathbf{2}\left(\mathrm{H} \mathrm{Cl} \cdot \mathrm{Au} \mathrm{Cl}^{3}\right) \\ \mathrm{C} & - & 26,8 & 26,31 & 35,24 \\ \mathrm{H} & - & 4,32 & 4,32 & 26,90 \\ & & & & 4,12\end{array}$

Einwirkung von Phosphorpentachlorid und Phosphoroxychlorid auf freies Lupinin.

Wenn, wie die Acetylierung dies beweist, in dem Lupinin zwei alkoholische Hydroxylgruppen enthalten sind, so liegt die Vermutung nahe, dass auch ein Ersatz der Hydroxylgruppen durch Chlor, wie beispielsweise beim Codein, möglich sein muss. Zur Prüfung dieser Annahme unternahm ich dje folgenden Versuche.

In einem trocknen Erlenmeyerschen Kolben wurde $1 \mathrm{~g}$ Phosphorpentachlorid mit $5 \mathrm{~g}$ Chloroform in Lösung gebracht und, unter $\mathrm{Ab}$ kühlung, $1 \mathrm{~g}$ fein zerriebenes Lupinin allmählich eingetragen. Nach 24stündigem Stehen in der Kälte wurde die klare, schwach gelbliche Lösung in Wasser gegossen, wodurch das Chloroform sich zuerst intensiv trübte, dann aber durch kräftiges Umschütteln wieder klärte. Da, 
nach dem Verdunsten des Chloroforms, kein Ruckstand blieb, musste die ganze Menge des eventuell gebildeten Körpers in der wässrigen Flüssigkeit sich befinden. Die beiden Flüssigkeiten wurden nun im Scheidetrichter getrennt, die wässrige Lösung etwas eingedampft und mit Goldchloridlösung versetzt, worauf ein dicker, alsbald flockig erscheinender Niederschlag sich ausschied. Einen Teil desselben krystallisierte ich aus salzsäurehaltigem Wasser um und erhielt dabei feine Krystallnädelchen. Dieselben zeigten bei $105^{\circ}$ keine Gewichtsabnahme und schmolzen bei $195^{\circ} \mathrm{zu}$ einem gelben Liquidum.

I. $0,189 \mathrm{~g}$ lufttrockner Substanz gaben $0,0720 \mathrm{~g} \mathrm{Au}$.

II. $0,2646 \mathrm{~g}$ gaben $0,1014 \mathrm{~g} \mathrm{Au}$.

III. $0,2411 \mathrm{~g}$ gaben $0,0914 \mathrm{~g} \mathrm{Au}$.

IV. $0,2717 \mathrm{~g}$ gaben bei der Elementaranalyse im Bleichromatrohre $0,2431 \mathrm{~g} \mathrm{CO}^{2}$ und $0,1018 \mathrm{~g} \mathrm{H} \mathrm{H}^{2} \mathrm{O}$.

\begin{tabular}{ccccc}
\multicolumn{5}{c}{ Gefundeu } \\
I. & II. & III. & IV. \\
$\mathrm{Au}$ & 38,09 & 38,32 & 37,9 & - \\
$\mathrm{C}$ & - & - & - & 24,40 \\
$\mathrm{H}$ & - & - & - & 4,16
\end{tabular}

Es lassen diese Daten nicht auf eine Einwirkung des Phosphorpentachlorids in dem gewünschten Sinn schliessen, sondern nur auf Lupiningoldchlorid.

Ich versuchte nunmehr auf anderem Wege zu dem erwähnten Chloro- bezw. Dichlorolupinid zu gelangen. Etwa $2 \mathrm{~g}$ Lupinin wurden drei Stunden lang am Rückflusskühler mit Phosphorpentachlorid und Phosphoroxychlorid im Ueberschuss erhitzt, die nach dem Erkalten gebliebene glasige, schwarzbraune Masse in wenig Wasser gelöst, die Lösung filtriert und das Filtrat mit Goldchloridlösung versetzt. Den sofort entstandenen käsigen Niederschlag filtrierte ich ab und krystallisierte einen Teil davon aus salzsäurehaltigem Wasser um. Die Krystalle trugen jedoch keinen einheitlichen Charakter; sie waren teils blättchenartig, teils nadelförmig ausgebildet. Von den nachstehenden Analysen sind die beiden ersten mit dem direkt ausgefällten, bei $100^{\circ}$ getrockneten Niederschlag, die übrigen mit dem umkrystallisierten Salz ausgeführt.

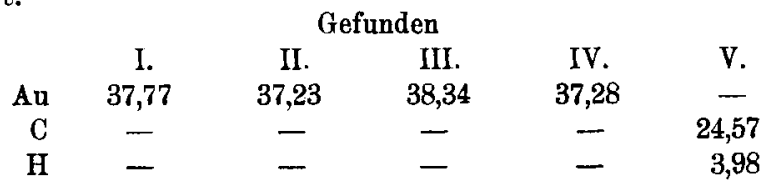

Diese Werte lassen kaum auf ein einheitliches Salz schliessen; es handelt sich hierbei anscheinend um ein Gemenge von: Lupiningoldchlorid, Monochlorolupinidgoldchlorid und Dichlorolupinidgoldchlorid. 
Bei einer weiteren Probe, wobei Phosphoroxychlorid in starkem Ueberschuss angewandt wurde, fiel auf Zusatz von Goldchloridlösung zu dem mit Wasser aufgenommenen Reaktionsprodukte, ein harzartig aussehender gelber Niederschlag aus, der nach dem Abfiltrieren, Auswaschen und Trocknen uber Schwefelsäure einen Schmp. von $38^{\circ}$ zeigte und dessen Analyse folgende Resultate gab:

I.

$\mathrm{Au} \quad 37,63$
II.

$\overrightarrow{32,80}$

Dieses Goldsalz war nur von geringer Beständigkeit; schon bei mehrtägigem Stehen über Aetzkalk verschwand die gleichmässig gelbe Farbe und machte einer braunen Platz. Aus der Mutterlauge dieses Golddoppelsalzes resultierten beim freiwilligen Verdunsten derselben uber Aetzkalk noch kleine, sternförmig gruppierte Krystalle, welche in ihrer Zusammensetzung dem Golddoppelsalze des gesuchten $\mathrm{Di}$ chlorolupinids entsprachen. Bei $100^{\circ}$ gaben dieselben kein Krystallwasser $\mathrm{ab}$; sie bräunten sich jedoch bei etwas höherer Temperatur.

I. $0,1996 \mathrm{~g}$ lufttrockener Substanz gaben $0,0739 \mathrm{~g} \mathrm{Au}$.

II. $0,1806 \mathrm{~g}$ gaben $0,238 \mathrm{~g} \mathrm{AgCl}$.

$\begin{array}{cccc} & \text { I. } & \text { II. } & \text { Berechnet für } \mathrm{C}^{21} \mathrm{H}^{88} \mathrm{~N}^{2} \mathrm{Cl}^{2,}, 2\left(\mathrm{H} \mathrm{Cl} \cdot \mathrm{Au} \mathrm{Cl}^{3}\right) \\ \mathrm{Au} & 36,87 & - & 36,70 \\ \mathrm{Cl} & - & 32,60 & 33,23\end{array}$

Nach den vorstehenden Beobachtungen kann es wohl keinem Zweifel unterliegen, dass bei der Einwirkung von Phosphorpentachlorid auf Lupinin die beiden OH-Gruppen dieser Base durch Chlor ersetzt werden. Das hierbei gebildete Dichlorolupinid: $\mathrm{C}^{21} \mathrm{H}^{38} \mathrm{~N}^{2} \mathrm{Cl}^{2}$, scheint jedoch nur von einer geringen Beständigkeit zu sein, es sind daher die Versuche, von dieser Verbindung ausgehend, zu dem Dianhydrolupinin, bezw. zu einer Base der Formel $\mathrm{C}^{21} \mathrm{H}^{40} \mathrm{~N}^{2}$ zu gelangen, vorläufig abgebrochen worden.

\section{Einwirkung von Methyljodid auf Lupinin und}

\section{Dianhydrolupinin.}

Aus dem Verhalten des Lupinins gegen Aethyljodid, welches Siewert sowohl, als auch Baumert ${ }^{1}$ ) bereits studierten, geht hervor, dass dieses Alkaloid eine tertiäre Base ist. Es schien mir von Interesse zu sein, auch das bezïgliche Verhalten gegen Methyljodid zu unter. suchen, um im Anschluss hieran das bisher nach dieser Richtung hin noch nicht geprüfte Dianhydrolupinin zu studieren. Zu diesem Zwecke

1) Habilitationschr. Halle 1881. 
löste ich zunächst $2 \mathrm{~g}$ Lupinin in etwas Methylalkohol, gab die dreibis vierfache Menge Jodmethyl zu und erhitzte diese Mischung etwa vier Stunden lang in einer Druckflasche im Dampf bade. Nach dem Erkalten erstarrte die Flüssigkeit gleichmässig zu einer weissen, krystallinischen Masse. Aus Alkobol umkrystallisiert, erhielt ich schön ausgebildete, anscheinend rhombische, wasserhelle Krystalle. Dieselben enthielten kein Krystallwasser.

I. $0,1850 \mathrm{~g}$ lufttrockener Substanz gaben $0,1374 \mathrm{~g} \mathrm{Ag} \mathrm{J}$.

II. $0,3100 \mathrm{~g}$ gaben $0,494 \mathrm{~g} \mathrm{CO}^{2}$ und $0,2121 \mathrm{~g} \mathrm{H}^{2} \mathrm{O}$.

III. $0,1681 \mathrm{~g}$ gaben $0,2659 \mathrm{~g} \mathrm{CO}^{2}$ und $0,1086 \mathrm{~g} \mathrm{H}^{2} \mathrm{O}$.

\begin{tabular}{ccccc} 
& \multicolumn{3}{c}{ Gefunden } & Berechnet für \\
& I. & II. & III. & $\mathrm{C}^{21} \mathrm{H}^{40} \mathrm{~N}^{2} \mathrm{O}^{2}\left(\mathrm{CH}^{3} \mathrm{~J}\right)^{2}$ \\
J & 39,83 & - & - & 39.93 \\
C & - & 43,45 & 7,58 & 43,39 \\
H & - & 43,13 & 7,17 & 7,23
\end{tabular}

Es hat somit auch bei der Einwirkung des Methyljodids auf Lupinin eine Addition von zwei Molekülen Jodmethyl stattgefunden, wie dies bereits durch Baumert für Jodaethyl nachgewiesen wurde.

Wie bei dem Lupinin, suchte ich auch einen Einblick in das Verhalten des Dianhydrolupinins bei der Einwirkung von Methyljodid $\mathrm{zu}$ gewinnen. Zu diesem Zwecke liess ich zunächst in der früher angegebenen Weise Phosphorsäureanhydrid auf freies Lupinin zirka 6 Stunden bei $200^{\circ}$ einwirken. Das Reaktionsprodukt, in wenig Wasser gelöst, wurde alsdann mit Natronhydrat alkalisch gemacht und die freie Base mit Aether ausgeschüttelt. Das nach dem Verdunsten des Aethers zurückgebliebene, gelbliche Liquidum, welches nicht in den krystallisierten Zustand übergeführt werden konnte, erwies sich durch das daraus dargestellte Goldsalz, als reines Dianhydrolupinin. Dasselbe brachte ich in eine Druckflasche, gab etwa die dreifache Menge Methyljodid zu und erhitzte das Gemisch sechs Stunden lang im Dampfbade. Das Einwirkungsprodukt zeigte sich nach dem Erkalten als eine braune, gleichmässige Flüssigkeit, die, nachdem sie zu einem dicken Extrakt eingedampft war, über Schwefelsäure und Aetzkalk auf bewahrt wurde. Da sich auch nach wochenlangem Stehen keine Krystallisation zeigte, löste ich die zähe Masse in verdünntem Alkohol, und erhitzte auf dem Lampf bade die Lösung solange mit Chlorsilber, bis alles Jod durch Silber gebunden war. Die filtrierte Flüssigkeit, auf ein kleines Volumen eingedampft, und mit Goldchloridlösung versetzt, ergab einen gelben, allmählich krystallinisch aussehenden Niederschlag.

Derselbe zeigte nach dem Trocknen einen Schmelzpunkt von $180^{\circ}$ und hatte kein Krystallwasser. 
I. $0,2458 \mathrm{~g}$ lufttrockener Substanz gaben $0,2411 \mathrm{~g} \mathrm{CO}^{2}$ und $0,0982 \mathrm{~g} \mathrm{H}^{2} \mathrm{O}$.

II. $0,2646 \mathrm{~g}$ gaben $0,1014 \mathrm{~g} \mathrm{Au}$.

III. $0,1666 \mathrm{~g}$ gaben $0,0645 \mathrm{~g} \mathrm{Au}$.

$\begin{array}{ccccc} & \text { I. } & \text { II. } & \text { III. } & \text { Ber. f. } \mathrm{C}^{21} \mathrm{H}^{36} \mathrm{~N}^{2}\left(\mathrm{CH}^{3}\right)^{2} 2\left(\mathrm{HCl} \cdot \mathrm{AuCl}^{3}\right) \\ \mathrm{Au} & - & 38,32 & 38,59 & 38,26 \\ \mathrm{C} & 26,74 & - & - & 26,87 \\ \mathrm{H} & 4,44 & - & - & 4,47 .\end{array}$

Diese Daten lehren, dass durch Einwirkung von Jodmethyl auf Dianhydrolupinin, "ebenso wie beim Lupinin, eine Addition von zwei Molekülen Jodmethyl stattgefunden hat. Es ist daher wahrscheinlich, dass auch das Dianhydrolupinin, ebenso wie das Lupinin, eine tertiäre Base ist.

\section{B. Das Lupinidin: $\mathrm{C}^{8} \mathrm{H}^{15} \mathrm{~N}$.}

Wie schon erwähnt, bediente ich mich bei der Darstellung der in den Samen der gelben Lupine enthaltenen beiden Alkaloide, insbesondere bei der Trennung derselben, zunächst der Baumert'schen Methode, welche auf der Unlöslichkeit des schwefelsauren Lupinidins in absolutem Alkohol beruht. Später schied ich jedoch das Lupinidin als in kaltem Wasser unlösliches Lupinidinquecksilberchlorid ab. Um zu letzterem Verfahren direkt die Rohalkaloidlösung verwenden zu können, ist es zweckmässig, daraus durch Bleiacetatlösung zuerst die Eiweissstoffe, sowie die sonstigen Verunreinigungen zu entfernen. Nach dem Entbleien durch Schwefelwasserstoff, sowie dem Verjagen des ersteren setzt man sofort Quecksilberchloridlösung im Ueberschuss zu. Das abgeschiedene Lupinidinquecksilberchlorid sammelt man auf einem Filter, suspendiert dasselbe in salzsäurehaltigem Wasser und sättigt die Mischung mit Schwefelwasserstoff. Alsdann wird das ausgefällte Quecksilbersulfid abfiltriert und das Filtrat, welches das Lupinin nunmehr als salzsaures Salz enthält, auf ein kleines Volumen eingedampft. Aus diesem Rückstande, den man hierauf mit Natronlauge alkalisch macht, schüttelt man schliesslich mit Aether die freie Base aus. Dieselbe stellt in diesem noch nicht vollkommen reinen Zustande eine gelbbraune, ölige Flüssigkeit dar. Säuert man dieses, noch unreine Lupinidin mit Salzsäure von neuem an und wiederholt in der oben erörterten Weise die Behandlung mit Quecksilberchlorid, so resultiert schliesslich das Alkaloid als schwach gelblich gefärbtes Liquidum. Die daraus hergestellten Salze trugen sämtlich den Stempel der Reinheit. Das reine Lupinidin, von oben erwähntem Aussehen, hat jedoch keineswegs, wie Liebscher und Baumert erwähnen, einen unangenehmen, schierlingsartigen Geruch, sondern erinnert in seinem Geruche, ebenso wie das Lupinin, an Fruchtäther. Allerdings verändert sich der Ge- 
ruch des Lupinidins allmählich, besonders, wenn es dem Licht und der Luft ausgesetzt wird, und nimmt dann die von Baumert erwähnten Eigenschaften an. Eine Krystallbildung bei dem Lupinidin, welche von Liebscher ${ }^{1}$ ) und Baumert ${ }^{2}$ ) erwähnt wird, konnte ich nicht konstatieren. Auch Baumert selbst konnte die erwähnte Beobachtung nur an der, von Liebscher inm zur Verfügung gestellten Probe machen.

Lupinidinsulfat: $\mathrm{C}^{8} \mathrm{H}^{15} \mathrm{~N} \cdot \mathrm{H}^{2} \mathrm{SO}^{4}$. Dieses bereits von Baumert analysierte Salz stellte ich aus dem durch Făllung mit Quecksilberchlorid erhaltenen, reinen Lupinidin derart her, dass ich letzteres mit überschüssiger, reiner Schwefelsäure (1:1) mischte und dann absoluten Alkohol in kleinen Portionen, unter kräftigem Umrïhren zugab. Sehr bald schied sich ein durchaus weisses Krystallmehl aus, welches, auf dem Saugfilter mit absolutem Alkohol von anhaftender Schwefelsäure befreit, zwischen Filtrierpapier und später über Schwefelsäure getrocknet wurde. Der Schmelzpunkt dieses Sulfats liess sich nicht bestimmen, da bei ca. $180^{\circ}$ eine Verkohlung des Salzes stattfand. Krystallwasser war nicht vorhanden.

I. 0,401 g lufttrockener Substanz gaben 0,4192 g Ba SO4.

II. $0,1966 \mathrm{~g}$ gaben $0,2061 \mathrm{~g} \mathrm{Ba} \mathrm{S0}$.

III. $0,3611 \mathrm{~g}$ gaben $0,5681 \mathrm{~g} \mathrm{CO}^{2}$ und $0,2562 \mathrm{~g} \mathrm{H}^{2}$.

$\begin{array}{ccccc} & \text { I. } & \text { II. } & \text { III. } & \text { Ber. f. } \mathrm{C}^{8} \mathrm{H}^{15} \mathrm{~N} \cdot \mathrm{H}^{2} \mathrm{SO}^{4} . \\ \mathrm{C} & - & - & 42,90 & 43,04 \\ \mathrm{H} & - & - & 7,88 & 7,62 \\ \mathrm{SO}^{3} & 35,79 & 35,99 & - & 35,87 .\end{array}$

Nach diesen Daten dürfte die dem vorliegenden Sulfat von Baumert zuerteilte Formel $\mathrm{C}^{8} \mathrm{H}^{15} \mathrm{~N} \cdot \mathrm{H}^{2} \mathrm{SO}^{4}$ wohl die richtige sein.

Lupinidinplatinchlorid: $\left(\mathrm{C}^{8} \mathrm{H}^{15} \mathrm{~N}\right)^{2} \cdot 2 \mathrm{HCl} \cdot \mathrm{PtCl}^{4}+2 \mathrm{H}^{2} \mathrm{O}$. Dieses Salz wird von den meisten der früheren Autoren als besonders charakteristisch für das Lupinidin bezeichnet. Ich erhielt dasselbe beim Versetzen der wässerigen Lösung des salzsauren Salzes mit Platinchloridlösung zunächst als hellgelben, sich schnell zu Boder setzenden Niederschlag, den ich alsdann aus heissem, salzsäurehaltigem Wasser umkrystallisierte. Die sich hierbei ausscheidenden Krystalle schienen dem rhombischen System anzugehören und waren von intensiv roter Farbe. Den Schmelzpunkt fand ich bisweilen bei $227^{\circ}$, doch zersetzte sich meist das Salz ohne vorheriges Schmelzen. Den Krystallwasserverlust fand ich den Angaben Baumerts durchaus entsprechend,

1) J. Kühns Ber. d. landw. Inst. Halle 270.

2) Lieb. Ann, 225. S. 365 u. ff. 
und zwar wird der Gewichtsverlust erst bei $135^{\circ}$ konstant. Eine Reihe von Analysen bestătigten weiter die Richtigkeit der Angaben obigen Autors.

I. $0,3640 \mathrm{~g}$ bei $135^{\circ}$ getrocknet, verloren $0,019 \mathrm{~g}$ an Gewicht; $0,3450 \mathrm{~g}$ wasserfreier Substanz gaben 0,1018 g Pt.

II. $0,2411 \mathrm{~g}$ verloren bei $13500,0125 \mathrm{~g} \mathrm{H} \mathrm{H}^{20} ; 0,2286 \mathrm{~g}$ wasserfreier Substanz gaben $0,0672 \mathrm{Pt}$.

III. $0,1966 \mathrm{~g}$ verloren bei $135^{0} 0,0102 \mathrm{~g} \mathrm{H} \mathrm{H}^{20} ; 0,1864 \mathrm{~g}$ wasserfreier Substanz gaben $0,0546 \mathrm{~g} \mathrm{Pt}$.

IV. $0,269 \mathrm{~g}$ wasserfreier Substanz gaben $0,2899 \mathrm{~g} \mathrm{CO}^{2}$ und $0,1188 \mathrm{~g} \mathrm{H}^{2}$.

V. $0,3121 \mathrm{~g}$ wasserfreier Substanz gaben $0,3351 \mathrm{~g} \mathrm{CO} 2$ und $0,1411 \mathrm{~g} \mathrm{H}^{2} \mathrm{O}$.

Diese Daten entsprechen im Mittel der Formel: $\left(\mathrm{C}^{8} \mathrm{H}^{15} \mathrm{~N}\right)^{2}$. $2 \mathrm{HCl} \cdot \mathrm{PtCl}^{4}+2 \mathrm{H}^{2} \mathrm{O}$.

\begin{tabular}{rcccccc}
\multicolumn{7}{c}{ Gefunden } \\
I. & II. & III. & IV. & V. & Berechnet \\
Pt & 29,50 & 29,39 & 29,29 & - & - & 29,49 \\
C & - & - & - & 29,39 & 29,31 & 29,11 \\
H & - & - & - & 4,90 & 4,70 & 4,85 \\
HH$^{20}$ & 5,22 & 5,18 & 5,18 & - & - & $5,20$.
\end{tabular}

Lupinidingoldehlorid: $\mathrm{C}^{8} \mathrm{H}^{15} \mathrm{~N} \cdot \mathrm{HCl} \cdot \mathrm{AuCl}^{8}$. Das Goldsalz dieser Base stellte ich her, indem ich eine salzsaure Lupinidinlösung mit Goldchlorid versetzte. Es entstand hierbei sofort ein gelber, voluminöser, alsbald käsig aussehender Niederschlag, der sich beim Erwärmen in salzsäurehaltigem Wasser leicht auflöste. Baumert ${ }^{1}$ ) hatte sich ebenfalls mit diesem Salz beschäftigt, doch trat nach seinen Angaben, beim Versuch, das Präparat umzukrystallisieren, unter Abscheidung von Gold, Zersetzung ein. Dies gelang mir jedoch zu vermeiden, indem ich beim Umkrystallisieren nur solange erwärmte, bis eben Lösung eingetreten war, dann rasch filtrierte und erkalten liess. Ich erhielt auf diese Weise das Salz in kleinen, gelben, nadelförmigen Kryställchen, die ich nach dem Trocknen zwischen Filtrierpapier, über Schwefelsäure aufbewahrte. Den Schmelzpunkt des Lupinidingoldchiorids fand ich bei $189^{\circ}$ liegend. Beim Versuch, den eventuellen Krystallwassergehalt $\mathrm{zu}$ bestimmen, zersetzte sich das Salz unter Abscheidung von Gold.

I. 0,2788 g lufttrockener Substanz gaben 0,1176 g Au.

II. 0,2111 g lufttrockener Substanz gaben 0,0896 g Au.

III. $0,2574 \mathrm{~g}$ lufttrockener Substanz gaben $0,1938 \mathrm{~g} \mathrm{CO}$ und 0,0866 $\mathrm{g} \mathrm{H}^{20}$.

IV. $0,3452 \mathrm{~g}$ gaben $0,2598 \mathrm{~g} \mathrm{CO}^{2}$ und $0,1112 \mathrm{~g} \mathrm{H}^{2} \mathrm{O}$.

I. II. III. IV. Ber. f. $\mathrm{C}^{8} \mathrm{H}^{15} \mathrm{~N} \cdot \mathrm{HCl} \cdot \mathrm{AuCl}^{3}$

$\begin{array}{rrrrrr}\mathrm{Au} & 42,18 & 42,44 & - & - & 42,30 \\ \mathrm{C} & - & - & 20,53 & 20,52 & 20,66 \\ \mathrm{H} & - & - & 3,73 & 3,57 & 3,65 .\end{array}$

1) Lieb. Ann. 225 S. 365 . 
Lupinidinquecksilberchlorid: $\left(\mathrm{C}^{8} \mathrm{H}^{15} \mathrm{~N}\right)^{2} \cdot 2 \mathrm{HCl} \cdot \mathrm{HgCl}^{2}$. Dieses sehr charakteristische und leicht zu reinigende Lupinidindoppelsalz schied sich beim Versetzen von Lupinidinhydrochloridlösung mit Quecksilberchloridlösung im Ueberschuss als ein weisser Krystallbrei ab, den ich zunächst auf einem Vakuumfilter sammelte und mit kaltem Wasser bis zur neutralen Reaktion auswusch. Derselbe erwies sich als fast unlöslich in kaltem, dagegen als löslich in heissem Wasser, woraus er als vollkommen gleichmässig weisses Krystallpulver resultierte. Bei $100^{\circ}$ zeigte das Salz keinen Gewichtsverlust, beim Erhitzen auf etwa $200^{\circ}$ zersetzte es sich unter Schwärzung.

I. $0,2710 \mathrm{~g}$ lufttrockenes Salz gaben $0,1058 \mathrm{~g} \mathrm{HgS}$ und $0,2628 \mathrm{~g} \mathrm{Ag} \mathrm{Cl}$.

II. $0,1891 \mathrm{~g}$ lufttrockener Substanz gaben $0,0737 \mathrm{~g} \mathrm{Hg} \mathrm{S}$ und $0,1916 \mathrm{~g} \mathrm{Ag} \mathrm{Cl}$.

I. II. Ber. f. $\left(\mathrm{C}^{8} \mathrm{H}^{15} \mathrm{~N}\right)^{2} \cdot 2 \mathrm{H} \mathrm{Cl} \cdot \mathrm{Hg} \mathrm{Cl}^{2}$

$\begin{array}{rrrr}\mathrm{Hg} & 33,65 & 33,80 & 33,67 \\ \mathrm{Cl} & 23,98 & 23,75 & 23,90\end{array}$

Demnach dürfte dem Lupinidinquecksilberchlorid die Formel: $\left(\mathrm{C}^{8} \mathrm{H}^{15} \mathrm{~N}\right)^{2} \cdot 2 \mathrm{H} \mathrm{Cl} \cdot \mathrm{Hg} \mathrm{Cl}^{2}$, zukommen.

Jodwasserstoffsaures Lupinidin: $\mathrm{C}^{8} \mathrm{H}^{15} \mathrm{~N} \cdot \mathrm{HJ}+1 / 2 \mathrm{H}^{2} \mathrm{O}$. Baumert ${ }^{1}$ ) erhielt dieses Salz als rötlich-weissen Niederschlag, als er eine konzentrierte -wässerige Lösung von salzsaurem Lupinidin mit einer kalt gesättigten Jodkaliumlösung versetzte. Aus heissem Wasser umkrystallisiert, schied sich das Salz dann in feinen, glänzenden Blättchen ab. Beim freiwilligen Verdunsten der Lösung im Vakuum, bildete das Salz Krystalle von alaunartigem Habitus, die zuerst farblos, durchsichtig waren, bald aber ganz undurchsichtig wurden. Nach erneutem Umkrystallisieren blieben jedoch diese Krystalle glashell. Die Analyse dieses Salzes führte Baumert zu der Formel: $\mathrm{C}^{8} \mathrm{H}^{15} \cdot \mathrm{HJ}$ $+1 / 2 \mathrm{H}^{2} \mathrm{O}$. Da mir die Darstellung eines jodwasserstoffsauren Salzes des Lupinidins von der Formel: $\left(\mathrm{C}^{8} \mathrm{H}^{15} \mathrm{~N}\right)^{2} \cdot \mathrm{HJ}$, gelungen war, versuchte ich auch das Hydrojodid Baumerts darzustellen, indem ich nach Angabe dieses Forschers schwefelsaures Lupinidin in konzentrierter wässeriger Lösung mit kalt gesättigter Jodkaliumlösung versetzte. Es entstand hierdurch ein gelbroter Niederschlag, der sich in heissem Wasser löste und nach dem Erkalten in Form glänzender Blättchen wieder abschied. Nach kurzer Zeit aber beobachtete ich die Bildung von Perjodiden; gleichzeitig schied sich Kaliumsulfat aus. Ich trennte daher die in Alkohol sich leicht lösenden Jodverbindungen von dem sich dabei abscheidenden Kaliumsulfat und erhielt, nach dem Verdunsten des Alkohols, in der That von neuem die obenerwähnten, blättchenförmigen Krystalle. Nach dieser Behandlung zersetzten sich

1) Lieb. Ann. S. 225, S. 375. 
dieselben jedoch, unter Bildung von Perjodiden, noch rascher als vorher, sodass eine Analyse derselben wenig aussichtsvoll erschien. Ich suchte daher zu diesem Hydrojodid zu gelangen, indem ich eine salz. saure Lupinidinlösung mit Jodkaliumlösung versetzte. Hierbei trat ebenfalls sofort eine gelblichweisse Ausscheidung auf, die aus heissem Wasser umkrystallisiert, schön ausgebildete, aneinandergelagerte, glänzendweisse Krystallblättchen lieferte. Die Analyse dieses Salzes ergab folgende Daten:

I. $0,3326 \mathrm{~g}$ verloren bei $100^{\circ} 0,0103 \mathrm{~g} \mathrm{H} \mathrm{H}^{2} \mathrm{O}, 0,3227 \mathrm{~g}$ wasserfreier Substanz gaben $0,301 \mathrm{lg} \mathrm{Ag} \mathrm{J}$.

II. $0,2112 \mathrm{~g}$ verloren bei $100^{\circ} 0,0064 \mathrm{~g} \mathrm{H} \mathrm{H}^{2} \mathrm{O} ; 0,2948$ wasserfreies Salz gaben $0,1899 \mathrm{~g} \mathrm{Ag} \mathrm{J}$.

$\begin{array}{rccc} & \text { I. } & \text { II. } & \text { Ber. f. } \mathrm{C}^{8} \mathrm{H}^{15} \mathrm{~N} \cdot \mathrm{HJ}+1 / 2 \mathrm{H}^{2} \mathrm{O} \\ \mathrm{J} & 50,44 & \mathbf{5 0 , 1 0} & 50,19 \% \\ \mathrm{H}^{2} \mathrm{O} & 30,06 & \mathbf{3 , 0 3} & \mathbf{3 , 0 3 \%}\end{array}$

Diese Werte stimmen mit den von Baumert für die Formel $\mathrm{C}^{8} \mathrm{H}^{15} \mathrm{~N} \cdot \mathrm{HJ}+1 / 2 \mathrm{H}^{2} \mathrm{O}$ gefundenen uberein.

Basisch jodwasserstoffsaures Lupinidin: $\left(\mathrm{C}^{8} \mathrm{H}^{15} \mathrm{~N}\right)^{2} \cdot \mathrm{HJ}$. Dieses Salz erhielt Baumert ${ }^{1}$ ) bei der Einwirkung von Aethyljodid auf freies Lupinidin; eine genaue Analyse desselben ist jedoch von diesem Forscher nicht ausgeführt worden. Mir gelang die Darstellung des fraglichen Jodids folgendermassen: Einen Teil des mit Aether ausgeschüttelten, sirupartigen Alkaloidgemisches, aus dem der grösste Teil des Lupinins bereits auskrystallisiert war, versetzte ich im Ueberschuss mit starker Jodwasserstoffsäure, rührte tüchtig durch, verdünnte mit Wasser und erhitzte diese Lösung auf dem Dampfbade. Nach dem Erkalten fand sich am Boden des Gefässes eine blättrige fest zusammenhängende Krystallmasse, die ich auf einem Saugfilter sammelte. Das Filtrat, auf ein kleines Volumen eingedampft, ergab nach dem Erkalten eine neue Menge dieses Salzes. Diese Krystalle erwiesen sich als in heissem Wasser leicht, in kaltem Wasser sehr schwer löslich. Nach dem Umkrystallisieren aus heissem Wasser erhielt ich das Salz in grossen blättrigen, zum Teil rosettenartig an einander gelagerten, undurchsichtigen Krystallen. Nach mehrmaligem Umkrystallisieren zeigte sich neben der ersten, eine zweite Krystallform in Gestalt durchsichtiger Rhomben. Später fand ich jedoch, dass in verdünnten Lösungen letztere, in konzentrierten dagegen erstere Form gebildet wurde. Beide Modifikationen zeigten denselben Schmelzpunkt: $230^{\circ}$, und erwiesen sich auch in ihrem übrigen Verhalten als vollkommen identisch. Ueber Schwefelsäure kurze Zeit

1) Lieb. Ann. 227 S. 207. 
getrocknet, hielten sie sich unverändert, auch bei $100^{\circ}$ getrocknet erlitten sie keine Gewichtsabnahme. Ich analysierte zuerst die blättchenförmigen, und dann die rhombischen Krystalle.

I. $0,1868 \mathrm{~g}$ lufttrockener Substanz gaben 0,1167 g AgJ.

II. $0,2002 \mathrm{~g}$ gaben $0,1244 \mathrm{~g} \mathrm{AgJ}$.

III. $0,2420 \mathrm{~g}$ gaben $0,1504 \mathrm{~g}$ AgJ.

IV. $0,1882 \mathrm{~g}$ gaben $0,1169 \mathrm{~g}$ AgJ.

V. $0,2861 \mathrm{~g}$ gaben im Bleichromatrohr mit vorgelegter Silberspirale verbrannt, $0,5364 \mathrm{~g} \mathrm{CO}^{2}$ und $0,2116 \mathrm{~g} \mathrm{H}^{2} \mathrm{O}$.

VI. $0,2051 \mathrm{~g}$ gaben $0,3841 \mathrm{~g} \mathrm{CO}^{2}$ und $0,1517 \mathrm{~g} \mathrm{H}^{20}$.

$\begin{array}{cccccccc} & \text { I. } & \text { II. } & \text { III. } & \text { IV. } & \text { V. } & \text { VI. Ber. f. }\left(\mathrm{C}^{8} \mathrm{H}^{15} \mathrm{~N}\right)^{2} \mathrm{HJ} \text {. } \\ \mathrm{J} & \mathbf{3 3 , 7 2} & 33,54 & 33,59 & \mathbf{3 3 , 5 5} & - & - & 33,59 \\ \mathrm{C} & - & - & - & - & 51,10 & 51,07 & 50,79 \\ \mathrm{H} & - & - & - & - & 8,76 & 8,21 & 8,20\end{array}$

Diese Daten dürften somit der Formel $\left(\mathrm{C}^{8} \mathrm{H}^{15} \mathrm{~N}\right)^{2} \mathrm{HJ}$, also einem basisch jodwasserstoffsauren Lupinidin entsprechen.

Dass es sich in der That um ein Lupinidinsalz handelte, bewies mir der Umstand, dass nach dem Umsetzen des Jodids mit Chlorsilber, durch Fällung mit Platinchloridlösung Lupinidinplatinchlorid resultierte, dessen Analyse folgendes Resultat ergab:

I. $0,1764 \mathrm{~g}$ verloren bei $135^{\circ} 0,0092 \mathrm{~g} \mathrm{H} \mathrm{H}^{2} \mathrm{O}$.

$0,1672 \mathrm{~g}$ wasserfreier Substanz gaben $0,0496 \mathrm{~g}$ entspr. $29,66_{o}^{\circ} \mathrm{Pt}$.

Gefunden Berechnet für $\left(\mathrm{C}^{8} \mathrm{H}^{15} \mathrm{~N}\right)^{2} \cdot 2 \mathrm{HCl} \cdot \mathrm{PtCl}^{4}+2 \mathrm{H}^{2} \mathrm{O}$

Pt 29,66 29,49

$\mathrm{H}^{20}$

5,21

5,17

Einwirkung von Jodalkyl auf freies Lupinidin.

Schon Siewert ${ }^{1}$ ) hatte versucht, durch Einwirkung von Jodäthyl auf das aus dem Samen der gelben Lupine isolierte „fluissige Basengemisch" bei $125^{\circ}$, einen Einblick in den chemischen Charakter des Lupinidins zu erhalten. Das Ergebnis der Siewertschen Versuche war: Eine Einwirkung scheint nicht stattgefunden zu haben. Der Röhreninhalt sonderte sich in zwei Schichten, eine schwere dunkelrote und eine leichte, schwach gelb gefärbte Flüssigkeit. Erstere erstarrte beim Abkühlen auf $10^{0}$ zu einer Krystallmasse, während die letztere nach dem Verdunsten einen in Aether löslichen Rückstand hinterliess, der stark alkalisch reagierte.

Baumert ${ }^{2}$ ), der, sich ebenfalls in einer Reihe von Versuchen mit obiger Frage eingehend beschäftigte, bestätigt die Angabe Siewerts. Er glaubte dabei die Thatsache beobachtet zu haben, dass das Alkaloid

1) Landw. Vers. Stat. 12, $306 u$ u. ff.

2) Lieb. Ann. 227. S. 207. 
mit Aethyljodid, selbst unter wechselnden Zeit- und Temperaturverhältnissen, stets nur teilweise damit in Reaktion tritt, so dass sich neben dem Einwirkungsprodukt, freies Aethyljodid, sowie freie Base vorfindet. Baumert fuhrte seine Versuche in einer Druckflasche aus, indem er anfänglich im Dampfbade erhitzte, später die Einwirkung, ca. 10 Stunden lang, in der Kälte vor sich gehen liess.

Das unveränderte Aethyljodid destillierte er ab, schüttelte die ausgeschiedene Krystallmasse mit alkoholfreiem Aether, behufs Entfernung des freien Lupinidins, welches er durch Darstellung des Platin. doppelsalzes als solches charakterisierte, und krystallisierte den festen Anteil des Reaktionsproduktes aus heissem Wasser um. Es resultierten hierbei wasserhelle Krystalle, aus denen sich durch Alkalihydrat, und darauf folgendes Ausschütteln mit Aether, reines Lupinidin isolieren liess, aus welchem wiederum Lupinidinplatinchlorid erhalten werden konnte. Bezüglich der Krystallform, beobachtete Baumert drei verschiedene Reaktionsprodukte.

1. Weisse, nadelförmige Krystalle,

2. eine weisse, blätterige Krystallmasse,

3. wasserhelle, wohlausgebildete Krystalle.

Aus drei Jodbestimmungen der erwähnten, verschiedenartigen Produkte, erhielt Baumert einen mittleren Jodgehalt von $35,35 \%$. Eine Elementaranalyse führte Baumert nicht aus, er suchte sich nur noch durch das Ausschütteln der freien Base zu vergewissern, ob thatsächlich Lupinidin oder ein Alkyladditionsprodukt desselben vorlag. Hierbei kam er dann zu dem Schluss, dass das betreffende, durch Alkalihydrat zersetzbare Produkt vielleicht ein basisch jodwasserstoffsaures Lupinidinsalz sei, welches in der Zusammensetzung der Formel $\left(\mathrm{C}^{8} \mathrm{H}^{15} \mathrm{~N}\right)^{2} \cdot \mathrm{HJ}$ entspricht. Dass es sich in der That im vorliegenden Fall um letzteres handele, glaubte Baumert noch aus dem Umstand folgern zu dürfen, dass der Jodgehalt von $35,35 \%$ sich nach jedem Umkrystallisieren der Substanz verminderte. Beim Eindampfen der Mutterlauge hatte Baumert ausserdem noch eine weissliche Krystallmasse erhalten, die durch Alkalihydrat nicht zerlegbar war und bei der Analyse Daten ergab, die auf ein äthyliertes Lupinin hinwiesen. Es handelte sich somit hierbei nur um ein Gemenge von Lupinin und Lupinidinverbindungen, wie auch ich dies bei dem ersten meiner diesbezüglichen Versuche, bei welchem kein vollständig einheitliches Lupinidin angewendet worden war, feststellen konnte. Da häufig Jodmethyl auf Alkaloide glatter reagiert als Jodäthyl, so verwendete ich ersteres, um die Frage nach dem Verhalten des Lupinidins gegen Jodalkyl zu entscheiden, und zwar erhitzte ich vollkommen reines, aus Lupinidinqueksilberchlorid dargestelltes Lupinidin in einer' 
Druckflasche mit einem Ueberschuss von Jodmethyl etwa drei Stunden lang im Dampfbad. Das Reaktionsprodukt erwies sich als eine gelbbraune Flüssigkeit; auf dem Dampfbade von überschüssigem Methyljodid befreit, erstarrte der grösste Teil der Masse nach dem Erkalten $\mathrm{zu}$ einem gelblichen Krystallbrei, den ich auf einem Vakuumfilter sammelte und mit Aether auswusch. Aus dem Filtrat erhielt ich nach dem Eindampfen nur noch einige Krystallfitter; auch nach dem rollständigen Verjagen des Lösungsmittels verblieb ein kaum bemerkenswerter Rückstand. Auch bei einem zweiten Versuch gelang es mir nioht die Beobachtung Baumerts zu bestatigen, dass bei der Einwirkung von Jodalkyl stets nur ein Teil des Lupinidins in Reaktion zu treten vermöge, dass also immer freies Lupinidin noch im Reaktions: produkt enthalten sei. Ich habe dies nur einmal beobachtet, als ich kein reines Lupinidin zur Anwendung brachte, wobei sich auch das Methyljodidderivat des Lupinins in dem Einwirkungsprodukt vorfand. Bei den mit reinem Materiale angestellten Versuchen erhielt ich in beiden Fällen, neben freiem Jodmethyl, ein vollkommen einseitliches, nur in der Krystallform verschiedenes Salz. Auch die letztere Verschiedenheit wurde hinfällig, nachdem ich durch die Analyse nachgewiesen hatte, dass das vorliegende Salz identisch mit dem von mir oben erwähnten basisch jodwasserstoffsauren Lupinidin ist, welches, wie dort erörtert, je nach der Konzentration des Lösungsmittels, verschiedene Krystallform zeigt.

Meiner Ansicht nach dürfte die Differenz in den Resultaten der Jodbestimmung, sowie auch die ubrigen Beobachtungen Baumerts, lediglich ihre Erklärung darin finden, dass dieser Forscher kein vollkommen reines Lupinidin zur Anwendung brachte. Von den folgenden Analysen ist I. mit blättchenförmigen, II. und III. mit rhombischen Krystallen ausgeführt.

I. $0,1821 \mathrm{~g}$ lufttrockener Substanz gaben $0,1121 \mathrm{~g} \mathrm{AgJ}$.

II. 0,1444 g lufttrockener Substanz gaben 0,0898 g AgJ.

III. 0,1992 g gaben bei der Elementaranalyse im Bleichromatrohre mit vorgelegter Silberspirale 0,3711 $\mathrm{CO}^{2}$ und $0,1542 \mathrm{~g} \mathrm{H}^{2} \mathrm{O}$.

$\begin{array}{ccccc} & \text { I. } & \text { II. } & \text { III. } & \text { Ber. f. }\left(\mathrm{C}^{8} \mathrm{H}^{15} \mathrm{~N}\right)^{2} \cdot \mathrm{HJ} \\ \mathrm{J} & 33,26 & 33,67 & - & 33,59 \\ \mathrm{C} & - & - & 50,80 & 50,79 \\ \mathrm{H} & - & - & 8,60 & 8,20\end{array}$

Um weiter die Ueberzeugung zu gewinnen, dass es sich im vorliegenden Falle in der That nur um ein Lupinidinsalz handele, setzte ich eine Probe davon mit Chlorsilber um und stellte dann aus dem salzsauren Filtrate das Platinsalz dar. Dasselbe erwies sich durch eine Wasser- und Platinbestimmung als vollkommen identisch mit Lupinidinplatinchlorid. 
$0,1488 \mathrm{~g}$ verloren bei $13500,0078 \mathrm{~g} \mathrm{H} \mathrm{H}^{2} \mathrm{O}$ entspr. $5,23 \% \mathrm{H}^{2} \mathrm{O}$.

$0,1410 \mathrm{~g}$ wasserfreier Substanz gaben 0,0414 g entspr. 29,36 \% Pt.

$\begin{array}{rcc} & \text { Gefunden } & \text { Berechnet für }\left(\mathrm{C}^{8} \mathrm{H}^{18} \mathrm{~N}\right)^{2} \cdot 2 \mathrm{H} \mathrm{Cl} \cdot \mathrm{PtCl}^{4}+2 \mathrm{H}^{2} \mathrm{O} . \\ \mathrm{H}^{20} & 5,23 & 5,17 \\ \mathrm{Pt} & 29,36 & 29,49 .\end{array}$

Es hat demnach die Einwirkung von Methyljodid auf das Lupinidin kein Produkt ergeben, welches Aufschluss über die Frage, ob dieses Alkaloid eine primäre, sekundäre oder tertiäre Base ist, geben konnte.

Ein Versuch, das Lupinidin durch Einwirkung von Brom zu bromieren, bezw. zu spalten, führte zu keinem Resultat. Das in der Druckflasche, nach dem Erhitzen von alkoholischer Bromlösung mit reinem Lupinidin, erhaltene Reaktionsprodukt ergab nach dem Umsetzen mit Chlorsilber durch Platinchloridlösung nur Lupinidinchloroplatinat, wie folgende Wasser- und Platinbestimmung zeigt:

$0,1864 \mathrm{~g}$ gaben bei $135^{0} 0,0096 \mathrm{~g}$ entspr. $5,15 \% \mathrm{H}^{2} 0$ Verlust.

$0,0519 \mathrm{~g}$ wasserfreier Substanz gaben 0,0519 g entspr. $29,35 \% \mathrm{Pt}$.

$\begin{array}{rcc} & \text { Gefunden } & \text { Berechnet für }\left(\mathrm{C}^{8} \mathrm{H}^{15} \mathrm{~N}\right)^{2} \cdot 2 \mathrm{H} \mathrm{Cl} \cdot \mathrm{PtCl}^{4}+2 \mathrm{H}^{2} \mathrm{O} \\ \mathrm{H}^{2} \mathrm{O} & 5,15 & 5,17 \\ \mathrm{Pt} & 29,35 & 29,49 .\end{array}$

Eine Einwirkung des Broms auf das Lupinidin war somit nicht erfolgt.

Das eigentümliche Verhalten des Lupinidins, ein einfach jodwasserstoffsaures Salz: $\mathrm{C}^{8} \mathrm{H}^{15} \mathrm{~N} \cdot \mathrm{HJ}+1 / 2 \mathrm{HO}$, sowie ein basisches Jodid: $\left(\mathrm{C}^{8} \mathrm{H}^{15} \mathrm{~N}\right)^{2} \cdot \mathrm{HJ}$, zu bilden, legten die Vermutung nahe, dass dem Lupinidin nicht die einfache Formel $\mathrm{C}^{8} \mathrm{H}^{15} \mathrm{~N}$, sondern die doppelte $\mathrm{C}^{16} \mathrm{H}^{30} \mathrm{~N}^{2}$, zukomme. Zur Aufklärung dieser wichtigen Frage, führte ich nach dem Raoult'schen Verfahren mittelst des Beckman'n'schen Apparates einige Bestimmungen der Molekulargrösse des einfach jodwasserstoffsauren Lupinidins $\mathrm{C}^{8} \mathrm{H}^{15} \mathrm{~N} \cdot \mathrm{HJ}+1 / 2 \mathrm{H}^{2} \mathrm{O}$ aus. Als Lösungsmittel benutzte ich Wasser und zwar 16,531 g. Verwendet wurden $0,1220 \mathrm{~g}$ jodwasserstoffsaures Lupinidin. Die molekulare Depression betrug 0,060 . Hieraus berechnet sich nach der Formel $m=\frac{c \cdot p}{\Delta \cdot 1}$, wo c eine konstante (für Wasser 19), $\mathrm{p}$ die Menge der angewendeten Substanz, $\Delta$ die mol. Depression, 1 die Menge des Lösungsmittels ist, die Molekulargrösse $=\frac{19 \cdot 0,122}{0,06 \cdot 16,5319} \cdot 100=233$. Bei einer zweiten Bestimmung kamen 0,4095 g des Jodids und 21,009 g Wasser zur Anwendung. Die Depression betrug 0,18; demnach die Molekulargrösse $=\frac{19 \cdot 0,4095}{0,18 \cdot 21,009} \cdot 100=257$. Da für die Formel $\mathrm{C}^{8} \mathrm{H}^{16} \mathrm{~N} \cdot \mathrm{HJ}+1 / 2 \mathrm{H}^{2} \mathrm{O}$ das Molekulargewicht $=261,5$ ist, so dürfte nach obigen Bestimmungen diese Formel die richtige sein. Durch die Feststellung dieser That- 
sache, sowie durch die Analysen der beiden jodwasserstoffisauren Salze, dirfte somit auch endgultig bewiesen sein, dass das Lupinidin eine sauerstofffreie Base ist und ihm die einfache, empirische Formel: $\mathrm{C}^{8} \mathrm{H}^{16} \mathrm{~N}$, zukommt. Die gleiche empirische Zusammensetzung kommt noch einigen dem Coniin nahestehenden Basen $\mathrm{zu}$, wie beispielsweise dem "Paraconiin" sowie den verschiedenen "Conicelnen". Soweit es sich nach den vorliegenden Litteraturangaben beurteilen lässt, ist jedoch das Lupinidin mit keiner dieser Basen identisch. Ob irgend welche nähere Beziehungen zwischen denselben obwalten, mag zunächst dahingestellt bleiben. Die Oxydationsversuche, welche ich mit Baryumpermanganat in alkalischer und in saurer Lösung ausfuhrte, haben bisher hierfür keinen Anhalt geliefert.

Bezüglich des Verhaltens des Lupinidins, bezw. dessen Salzen gegen den polarisierten Lichtstrahl ist zu bemerken, dass die von mir untersuchten Salze: das schwefelsaure Lupinidin, das salzsaure Lupinidin, das jodwasserstoffsaure Lupinidin alle denselben stark nach links ablenken.

\section{Mitteilungen aus der pharmazeutischen Abteilung des}

Eidgenössischen Polytechnikum in Zürich.

\section{Ueber die Bisabol-Myrrha.}

Von W. Tucholka.

(Eingegangen den 13. V. 1897.)

Neben der offizinellen Myrrha, die in Arabien und in Afrika in den Küstengegenden der Somaliländer von mehreren CommiphoraArten gesammelt wird ${ }^{1}$, existiert noch eine andere, vielleicht sogar noch mehrere Sorten. Ueber diese zweite Sorte, die bei den Somali

1) Schweinfurth, Ber. d. pharm. Ges. 1893, p. 218.

F'lückiger, Pharmakognosie 3. Autl., p. 43. 